\title{
Antihistamine effects and safety of fexofenadine: a systematic review and Meta-analysis of randomized controlled trials
}

Cheng-zhi Huang ${ }^{1,2+}$, Zhi-hui Jiang ${ }^{3+}$, Jian Wang ${ }^{1}$, Yue Luo ${ }^{4,5}$ and Hua Peng ${ }^{1 *}$ (D)

\begin{abstract}
Background: As a new generation antihistamine, fexofenadine has been widely used in allergic diseases. However, there is still a lack of collective evidence regarding the antihistamine effects and safety profiles of fexofenadine relative to other antihistamine drugs and placebo. Therefore, we aimed to systematically evaluate the antihistamine effects and safety of fexofenadine.

Methods: An electronic literature search of randomized controlled trials (RCTs) was performed using Embase, Cochrane and PubMed from establishment to January 1st, 2018. RCTs comparing the antihistamine effects or safety (adverse events, sedative effects, and cognitive/psychomotor function) of fexofenadine with either other antihistamines or placebo for healthy subjects and patients with allergy were selected.

Results: Fifty-one studies of 14,551 participants met the inclusion criteria. When compared with the first-generation antihistamines, fexofenadine produced significantly lower adverse events frequency $(\mathrm{OR}=0.446 ; 95 \% \mathrm{Cl}: 0.214$ to $0.929, P=0.031)$, significantly lower sedative effects frequency $(\mathrm{OR}=0.265 ; 95 \% \mathrm{Cl}: 0.072$ to $0.976, P=0.046)$ and significantly less change of all cognitive/psychomotor function. When compared with the second-generation antihistamines, fexofenadine produced significantly marginal sedative effects $(\mathrm{OR}=0.59 ; 95 \% \mathrm{Cl}, 0.38$ to $0.93 ; P=$ 0.02 ) and significantly less change of most of the cognitive/psychomotor function. When compared with placebo, fexofenadine produced more significant antihistamine effects.

Conclusions: Fexofenadine has a positive antihistamine effect, which is probably no worse than the secondgeneration antihistamines. Fexofenadine probably has a favorable safety profile, which is more likely better than that of the first-generation antihistamines. There is lack of data to support that fexofenadine has a better overall safety profile compared to the second-generation antihistamines, however, some presently available evidence on sedative effects and certain aspects of cognitive/psychomotor function favors fexofenadine. Therefore, fexofenadine may be worthy of recommendation for safety related workers.
\end{abstract}

Keywords: Fexofenadine, Antihistamines, Randomized controlled trial, Systematic review, Meta-analysis

\footnotetext{
* Correspondence: doctorpeng2006@hotmail.com

${ }^{+}$Cheng-zhi Huang and Zhi-hui Jiang contributed equally to this work

${ }^{1}$ Department of Otolaryngology Head and Neck Surgery, General Hospital of

Southern Theatre Command of PLA, Guangzhou 510010, China

Full list of author information is available at the end of the article
}

(c) The Author(s). 2019 Open Access This article is distributed under the terms of the Creative Commons Attribution 4.0 International License (http://creativecommons.org/licenses/by/4.0/), which permits unrestricted use, distribution, and reproduction in any medium, provided you give appropriate credit to the original author(s) and the source, provide a link to the Creative Commons license, and indicate if changes were made. The Creative Commons Public Domain Dedication waiver (http://creativecommons.org/publicdomain/zero/1.0/) applies to the data made available in this article, unless otherwise stated. 


\section{Background}

The incidence of allergic diseases such as allergic rhinitis (AR), allergic asthma (AA), chronic idiopathic urticaria $(\mathrm{CIU})$ and atopic dermatitis (AD) has continued to rise over the past several decades, affecting a large number of people worldwide [1]. Symptoms such as itching, sneezing, rhinorrhea and rhinobyon caused by allergic diseases usually lower the quality of life [2]. In fact, millions of people have been reported to experience physical impairments and reductions in quality of life, as well as economic burdens, derived from allergic diseases and its associated comorbidities [3]. Antihistamines have been widely used as a first-line drug in the treatment of allergic diseases. The first-generation antihistamines were no longer recommended because of their side effects including hepatotoxicity, cardiotoxicity, sedative effects, anticholinergic effects and lack of selectivity for the H1-receptor [4]. The second-generation antihistamines have replaced the firstgeneration antihistamines as commonly used drug in the treatment of allergic diseases because of their modest sedative effects and more significant and persistent curative effect compared with the first-generation antihistamines [4]. However, some of the second-generation antihistamines, such as terfenadine and astemizole, are rarely used because of their apparent cardiotoxicity [5]. As a new generation antihistamine and an active metabolite of terfenadine - a highly selective H1 antagonist, fexofenadine has positive antihistamine effects [6]. In addition, fexofenadine has no cardiotoxicity and minimal adverse effects on liver because only about $5 \%$ dosage of fexofenadine is metabolized by liver. As the substrate of Pglycoprotein, fexofenadine that is difficult to pass the blood-brain barrier may have no sedative effect and other central nervous functions [7]. To date, there is still a lack of collective evidence regarding the antihistamine effects and safety profiles of fexofenadine relative to other antihistamine drugs and placebo. As such, the aim of this study was to analyze the antihistamine effects and safety of fexofenadine in healthy subjects and patients with allergic diseases including $\mathrm{AR}, \mathrm{AA}, \mathrm{CIU}$, and $\mathrm{AD}$ when compared with other antihistamines or placebo.

\section{Methods}

\section{Eligibility criteria}

Randomized controlled trials (RCTs) involving comparisons of antihistamine effects and safety of fexofenadine with either other antihistamines or placebo were included. Participants in these RCTs including healthy volunteers and patients with indications requiring treatment of antihistamines.

\section{Search strategy}

A systematic literature search of Embase, Cochrane and PubMed were conducted with no limits on language, publication year, or publication status. The date of the last search was January 1st, 2018. The search term strategy was as follows: "fexofenadine", "telfast", "allegra", AND "health*", "allerg*", "rhinitis", “cold", "asthma", "Kimura”, "atopic”, “dermatitis”, "atopy”, "urticaria”, OR "effec*", "antihistami*", "skin", "wheal”, "flare", "safe ${ }^{*}$ ", "drows"*, "sleep*", "somnolence", "alert*", and "sedat". References of included studies and additional sources were examined to reduce the search bias.

\section{Study selection process}

Endnote X7 program was used to eliminate duplicate references. The first round of screening was performed by reading title and abstract, the second round of screening was eligibility evaluation from the full text. All operations were performed by 2 separate reviewers and checked by the principal investigator. Any disagreements were resolved by discussion.

\section{Data extraction}

For each included literature, the following data were extracted: first author, date of publication, mean age, gender, number of subjects lost to follow-up, study type, participant, number of subjects receiving fexofenadine, comparators, number of subjects receiving comparators, the dose of fexofenadine and comparators, study duration, outcome measures. If more than 1 dose of fexofenadine or more than 1 type of other generation antihistamines were assessed, we selected the one considered more effective and safer by the authors of the paper as the assessment of antihistamine effects and cognitive/psychomotor function, and combined all doses of fexofenadine or the same generation antihistamines for the evaluation of adverse events (AE) frequency and sedative effects frequency. When data were not available in certain papers, the authors were contacted directly by e-mail. If the results were only presented in graphs, these were digitalized and then converted to numbers using the Digitizelt 1.5.7 program (Digitizelt 2003; Bormann, Braunschweig, Germany). Two independent reviewers extracted data from the selected papers, reconciling differences by consensus.

The outcomes measured were as follows: antihistamine effects were assessed by the inhibition rate of histamine-induced wheal and flare (24 h after treatment); safety was assessed by AE frequency, sedative effects frequency and the change of cognitive/psychomotor function scores (3 to $5 \mathrm{~h}$ after treatment). Cognitive/ psychomotor function scores included critical flicker fusion (CFF), choice reaction time (CRT), compensatory tracking test (CTT), line analogue rating scales for sedation (LARS), and visual analogue score (VAS) of drowsiness, which were used for the assessment of information processing capability, reaction speed, the 
degree of attention focusing, vigilance and fatigue, somnolence degree. Annotations of cognitive/psychomotor function scores are as follows:

CFF. The CFF referred to the frequency of intermittent light stimulation when the flicker happened to achieve fusion and was used to evaluate the information processing ability. Our eyes will produce a sense of flicker when receiving light stimulation with low intermittent frequency. With a gradual increase of the intermittent frequency produced by light stimulation, the flicker gradually disappears. Our eyes will feel a steady and continuous light when the flicker reaches a certain frequency, which is called the fusion of flicker. The decrease in CFF suggested a reduction in the ability to process information.

CRT. The CRT was taken as a sensitive measurement of drug-induced changes in psychomotor speed. From a central starting position subjects were required to extinguish one of the six red lights and illuminated at random by touching the appropriate response button. The increase in CRT indicated a reduction in the response speed of subjects.

CTT. The CTT was used as a means to assess divided attention. Subjects were required to keep a cursor in alignment with a moving target on a visual display unit screen using a mouse. The evaluation measure of this tracking task was the mean difference between the centers of the target and cursor in pixels, sampled 5 times per second, during the 10 min test period. Higher scores were indicative of less concentration.

LARS. The LARS was employed as a measure of the subjective effects of psychoactive drugs. Subjects marked a series of $10 \mathrm{~cm}$ line analogue scales, indicating their present feeling with regards to a mid-point, which represented their normal state of mind before treatment began. The mean scores of ratings of 'tiredness', 'drowsiness', and 'alertness', presented among several distracter scales, were taken as a measurement of perceived sedation. The higher the score (in $\mathrm{mm}$ ), the less alert and more tired and drowsy the subjects felt.

VAS of drowsiness. The VAS of drowsiness was used as a subjective indicator of somnolence degree marking by subjects with end points of 'not drowsy' and 'very drowsy'. Higher scores were indicative of more somnolent.

\section{Risk of Bias assessment}

The risk of bias and methodological quality were evaluated using the Cochrane Collaboration tool [8]. There are 6 aspects including (a) sequence generation, (b) allocation concealment, (c) blinding of caregivers, personnel and outcome assessors, (d) incomplete outcome data, (e) selective outcome reporting, and (f) other sources of bias need to be graded as three levels of risk: (A) low risk of bias, (B) unclear risk of bias, and (C) high risk of bias. Two independent reviewers assessed the risk of bias of the selected studies, reconciling differences by consensus.

\section{Data synthesis}

Data analysis was performed using the RevMan 5.3 program (The Cochrane Collaboration, Oxford, UK) and Comprehensive Meta Analysis V2 (Biostat, Englewood, NJ 07631 USA). Pooled weighted mean differences (WMDs) was used for continuous data (the inhibition rate of histamine-induced wheal and flare, the change of cognitive/ psychomotor function scores). Odds ratio (OR) was used for dichotomous data (AE frequency, sedative effects frequency). Heterogeneity was assessed by $I^{2}$ and Cochrane's $\mathrm{Q}$ test. When heterogeneity was not present $\left(I^{2}<50 \%\right)$, fixed-effects model (FEM) and Peto OR were applied. For $I^{2}>50 \%$, a random-effects model (REM) and DerSimonianLair OR were used. Potential publication bias was evaluated using funnel plots. Sensitivity analysis was performed by eliminating the selected studies one by one.

\section{Results}

\section{Search results}

As shown in Fig. 1, our search identified 841 records; 394 were excluded due to duplication, 351 were excluded after the first round of screening, and 96 full-text articles were assessed for eligibility. Of these, 25 were excluded because they were reviews, case reports, open studies, or studies aimed at other purposes. Seventy-one clinical trials on the comparison of antihistamine effects or safety of fexofenadine with other antihistamines or placebo for participants were potentially relevant. The second round of screening excluded 20 comparative trials which had outcomes not eligible for inclusion criteria. Finally, 51 RCTs satisfied the inclusion criteria and then were included in our meta-analysis [9-59]. Notably, partial data from 8 RCTs $[15,24,28,30,36,53,56,58]$ were only reported in graphics and; the attempt to obtain data directly from the authors failed, so graphics were digitized and the SD were estimated using an imputation method.

\section{Trial characteristics}

Of 51 included studies, $20(39.2 \%)$ are parallel and 31 $(60.8 \%)$ are cross-over trials. The mean age of a total of 14,551 participants included was 31.7 years and $45.0 \%$ were male. Among these participants were 3024 cases of healthy subjects, 10,521 cases of AR, 536 cases of CIU, 470 cases of pollinator. Characteristics of the included studies are shown in Table 1. 


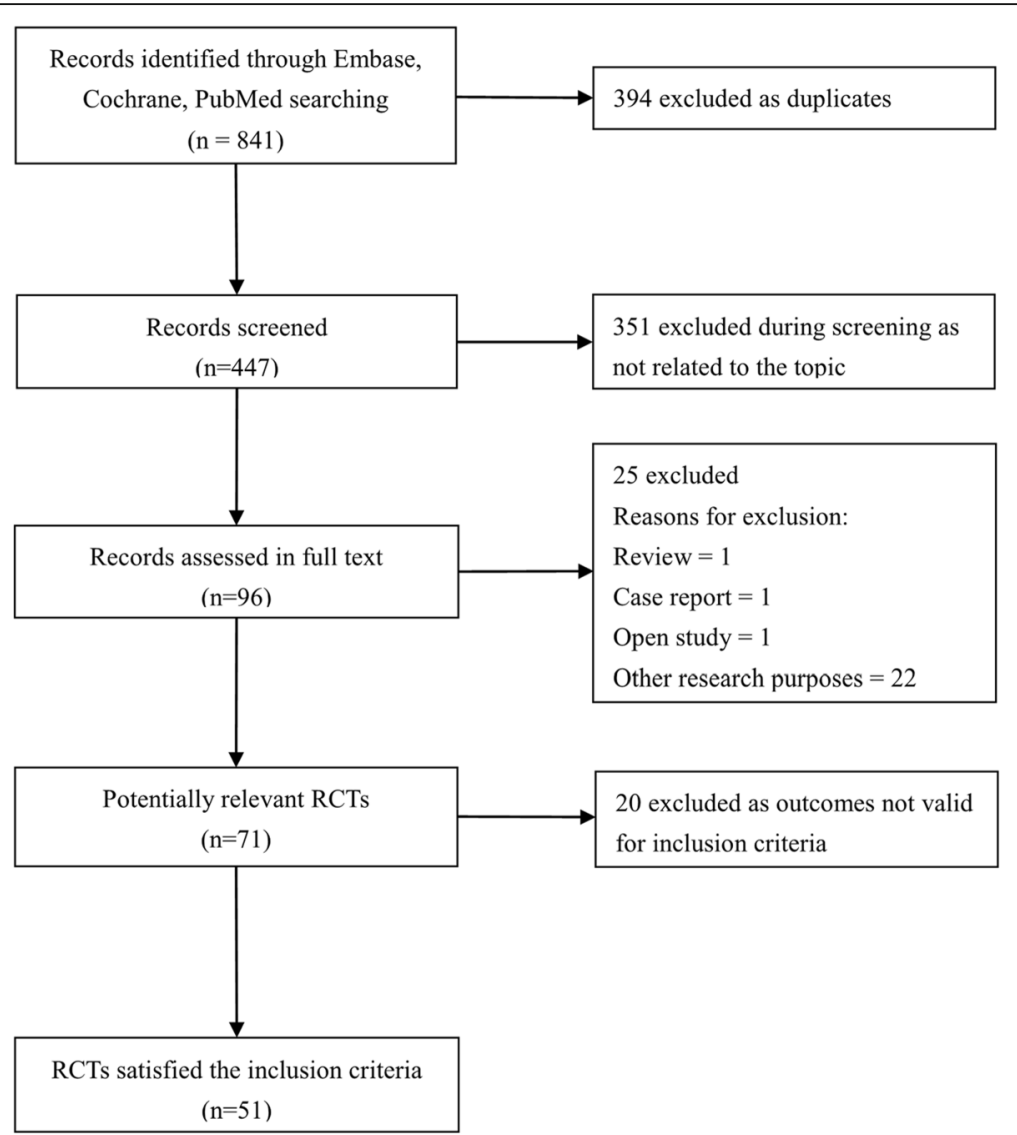

Fig. 1 Flow diagram of study selection

\section{Antihistamine effects}

\section{The inhibition rate of histamine-induced wheal}

Six studies reported the inhibition rate of histamineinduced wheal after taking fexofenadine [25, 31, 36, 5557]. Of the 6 studies on healthy subjects, 1 compared with the first-generation antihistamines [55], 5 compared with the second-generation antihistamines [25, 31, 36, $55,56]$, and 5 compared with placebo [25, 31, 36, 56, 57]. The comparison between fexofenadine and the firstgeneration antihistamines was not pooled for metaanalysis because there was only 1 study included. When compared with the second-generation antihistamines, as shown in Fig. 2, the inhibition rates of histamineinduced wheal were not different (WMD $=-17.56 ; 95 \%$ CI: -44.77 to $9.65, P=0.21$ ). The heterogeneity was $99 \%$, which may be generated from the inconsistent doses of fexofenadine and types of the secondgeneration antihistamines. When compared with placebo, the results indicated that fexofenadine produced significantly higher inhibition rate of histamine-induced wheal. After sensitivity analysis and checking the trial methods, 2 studies were excluded because of different study duration compared with other studies [25, 36]. As shown in Additional file 2: Figure S2 the inhibition rate of histamine-induced wheal of fexofenadine was significantly higher than that of placebo (WMD $=-18.93 ; 95 \%$ CI: 15.29 to $22.57, P<0.00001)$. A $I^{2}$ of $8 \%$ represents low heterogeneity.

\section{The inhibition rate of histamine-induced flare}

Six studies reported the inhibition rate of histamineinduced flare after taking fexofenadine [25, 31, 36, 5557]. Of the 6 studies on healthy subjects, only 1 compared with the first-generation antihistamines [55], which was not suitable for meta-analysis. Four studies compared with the second-generation antihistamines $[25,36,55,56]$, as shown in Fig. 3, the inhibition rate of histamine-induced flare were not different (WMD = 4.58; $95 \%$ CI -40.70 to $49.85, P=0.84)$. Five studies compared with placebo [25, 31, 36, 56, 57], as shown in Additional file 3: Figure S3, fexofenadine produced significantly higher inhibition rate of histamine-induced flare $(\mathrm{WMD}=35.75,95 \% \mathrm{CI}: 18.67$ to $52.83, \quad P<$ $0.00001)$. The heterogeneity may be generated from the inconsistent doses of fexofenadine and different type of the second-generation antihistamines. Sensitivity analysis showed the meta-analysis results were similar. 
Table 1 Characteristics of included studies

\begin{tabular}{|c|c|c|c|c|c|c|c|}
\hline First author, Years & Study design & Subject & Number & Experimental & Comparators & $\begin{array}{l}\text { Study } \\
\text { duration }\end{array}$ & Outcome measures \\
\hline Barbanoj [25], 2003 & $\begin{array}{l}\text { RCT, } \\
\text { crossover } \\
\text { study }\end{array}$ & healthy & 18 & $\begin{array}{l}\text { FEX } 120 \mathrm{mg} \\
n=18\end{array}$ & $\begin{array}{l}\text { EBA } 20 \mathrm{mg} . \\
n=18 \\
\mathrm{PL} n=18\end{array}$ & 5 days & $\begin{array}{l}\text { Wheal, flare, } A E \text {, sedative } \\
\text { effects }\end{array}$ \\
\hline Boyle [36], 2005 & $\begin{array}{l}\text { RCT, } \\
\text { crossover } \\
\text { study }\end{array}$ & healthy & 18 & $\begin{array}{l}\text { FEX } 60 \mathrm{mg} \\
n=18\end{array}$ & $\begin{array}{l}\text { LOR } 10 \mathrm{mg} \\
n=18 \\
\text { PL } n=18\end{array}$ & 2 months & $\begin{array}{l}\text { Wheal, flare, AE, sedative } \\
\text { effects }\end{array}$ \\
\hline Larbig [57], 2006 & $\begin{array}{l}\text { RCT, } \\
\text { crossover } \\
\text { study }\end{array}$ & healthy & 30 & $\begin{array}{l}\text { FEX } 120 \mathrm{mg} \\
n=30\end{array}$ & $\mathrm{PL} n=30$ & $24 \mathrm{~h}$ & Wheal, flare \\
\hline Simons [55], 2002 & $\begin{array}{l}\text { RCT, parallel } \\
\text { study }\end{array}$ & healthy & 21 & $\begin{array}{l}\text { FEX } 180 \mathrm{mg} \\
n=7\end{array}$ & $\begin{array}{l}\text { CHL } 8 \mathrm{mg} \\
n=7 \\
\text { LOR } 10 \mathrm{mg} \\
n=7\end{array}$ & 9 days & Wheal, flare \\
\hline Simons [31], 2003 & $\begin{array}{l}\text { RCT, } \\
\text { crossover } \\
\text { study }\end{array}$ & healthy & 15 & $\begin{array}{l}\text { FEX } 30 \mathrm{mg} \\
n=15\end{array}$ & $\begin{array}{l}\text { CET } 10 \mathrm{mg} \\
n=15\end{array}$ & $24 \mathrm{~h}$ & $\begin{array}{l}\text { Wheal, flare, sedative } \\
\text { effects }\end{array}$ \\
\hline $\begin{array}{l}\text { Takahashi [56], } \\
2004\end{array}$ & $\begin{array}{l}\text { RCT, } \\
\text { crossover } \\
\text { study }\end{array}$ & healthy & 7 & $\begin{array}{l}\text { FEX } 120 \mathrm{mg} \\
n=7\end{array}$ & $\begin{array}{l}\text { BEP } 20 \mathrm{mg} \\
n=7 \\
\mathrm{PL} n=7\end{array}$ & $24 \mathrm{~h}$ & $\begin{array}{l}\text { Wheal, flare, VAS of } \\
\text { drowsiness }\end{array}$ \\
\hline Finn [11], 1999 & $\begin{array}{l}\text { RCT, parallel } \\
\text { study }\end{array}$ & $\mathrm{CIU}$ & 439 & $\begin{array}{l}\mathrm{FEX} 40 \mathrm{mg} / 120 \mathrm{mg} / 240 \\
\mathrm{mg} / 480 \mathrm{mg} \\
n=349\end{array}$ & $P L n=90$ & 4 weeks & $A E$, sedative effects \\
\hline Berger [41], 2006 & $\begin{array}{l}\text { RCT, parallel } \\
\text { study }\end{array}$ & AR & 432 & $\begin{array}{l}\text { FEX } 180 \mathrm{mg} \\
n=288\end{array}$ & $\mathrm{PL} n=144$ & 15 days & $A E$, sedative effects \\
\hline $\begin{array}{l}\text { Berkowitz [42], } \\
2006\end{array}$ & $\begin{array}{l}\text { RCT, } \\
\text { crossover } \\
\text { study }\end{array}$ & AR & 63 & $\begin{array}{l}\text { FEX } 180 \mathrm{mg} \\
n=63\end{array}$ & PL $n=63$ & 2 weeks & $A E$, sedative effects \\
\hline Boyle [43], 2006 & $\begin{array}{l}\text { RCT, } \\
\text { crossover } \\
\text { study }\end{array}$ & healthy & 18 & $\begin{array}{l}\text { FEX } 120 \mathrm{mg} \\
n=18\end{array}$ & $\begin{array}{l}\text { CHL } 6 \mathrm{mg}, \mathrm{n}=18 \\
\mathrm{PL} n=18\end{array}$ & $10 \mathrm{~h}$ & $A E$, sedative effects \\
\hline Bronsky [10], 1998 & $\begin{array}{l}\text { RCT, parallel } \\
\text { study }\end{array}$ & AR & 548 & $\begin{array}{l}\text { FEX } 80 \mathrm{mg} / 120 \mathrm{mg} / \\
240 \mathrm{mg}, \\
n=411\end{array}$ & PL $n=137$ & 14 days & $A E$, sedative effects \\
\hline Casale [13], 1999 & $\begin{array}{l}\text { RCT, parallel } \\
\text { study }\end{array}$ & AR & 861 & $\begin{array}{l}\text { FEX } 120 \mathrm{mg} / 180 \mathrm{mg} \\
n=569\end{array}$ & PL $n=292$ & 3 weeks & $A E$, sedative effects \\
\hline $\begin{array}{l}\text { Van Cauwenberge } \\
{[17], 2000}\end{array}$ & $\begin{array}{l}\text { RCT, parallel } \\
\text { study }\end{array}$ & $A R$ & 685 & FEX $120 \mathrm{mg}, n=232$ & $\begin{array}{l}\text { LOR } 10 \text { mg, } \\
n=228 \\
\text { PL } n=225\end{array}$ & 14 days & $A E$, sedative effects \\
\hline Day [33], 2004 & $\begin{array}{l}\text { RCT, parallel } \\
\text { study }\end{array}$ & $A R$ & 575 & FEX $180 \mathrm{mg}, n=239$ & $\begin{array}{l}\text { CET } 10 \text { mg, } \\
n=240 \\
\text { PL } n=96\end{array}$ & $24 \mathrm{~h}$ & $A E$, sedative effects \\
\hline Ramesh [50], 2013 & $\begin{array}{l}\text { RCT, parallel } \\
\text { study }\end{array}$ & AR & 50 & FEX $120 \mathrm{mg}, n=25$ & $\begin{array}{l}\text { CHL } 4 \mathrm{mg}, \\
n=25\end{array}$ & 14 days & $A E$, sedative effects \\
\hline Grant [14], 1999 & $\begin{array}{l}\text { RCT, } \\
\text { crossover } \\
\text { study }\end{array}$ & healthy & 14 & $\begin{array}{l}\text { FEX } 60 \mathrm{mg} \\
n=14\end{array}$ & $\begin{array}{l}\text { CET } 10 \mathrm{mg} \\
n=14 ; \\
\text { EPI } 20 \mathrm{mg} \\
n=14 ; \\
\text { TER } 60 \mathrm{mg} \\
n=14 ; \\
\text { LOR } 10 \mathrm{mg} \\
n=14 \\
\text { PL } n=14\end{array}$ & $24 \mathrm{~h}$ & $A E$, sedative effects \\
\hline Grant [23], 2002 & $\begin{array}{l}\mathrm{RCT} \text {, } \\
\text { crossover } \\
\text { study }\end{array}$ & healthy & 18 & FEX $180 \mathrm{mg}, \mathrm{n}=18$ & $\begin{array}{l}\text { EBA } 10 \mathrm{mg} \\
n=18 \\
\text { LOR } 10 \mathrm{mg} \\
n=18 \\
\text { MIZ } 10 \mathrm{mg} \\
n=18\end{array}$ & $24 \mathrm{~h}$ & $A E$, sedative effects \\
\hline
\end{tabular}


Table 1 Characteristics of included studies (Continued)

\begin{tabular}{|c|c|c|c|c|c|c|c|}
\hline First author, Years & Study design & Subject & Number & Experimental & Comparators & $\begin{array}{l}\text { Study } \\
\text { duration }\end{array}$ & Outcome measures \\
\hline & & & & & $\mathrm{PL} n=18$ & & \\
\hline Hampel [26], 2003 & $\begin{array}{l}\text { RCT, parallel } \\
\text { study }\end{array}$ & $A R$ & 495 & FEX $180 \mathrm{mg}, n=248$ & CET $10 \mathrm{mg}, n=247$ & 2 weeks & $A E$, sedative effects \\
\hline Hampel [45], 2007 & $\begin{array}{l}\mathrm{RCT} \text {, parallel } \\
\text { study }\end{array}$ & $A R$ & 393 & $\begin{array}{l}\text { FEX } 30 \mathrm{mg} / 60 \mathrm{mg} \\
n=193\end{array}$ & $\mathrm{PL} n=200$ & 8 days & $A E$, sedative effects \\
\hline $\begin{array}{l}\text { Hashiguchi [52], } \\
2016\end{array}$ & $\begin{array}{l}\text { RCT, } \\
\text { crossover } \\
\text { study }\end{array}$ & healthy & 126 & FEX $120 \mathrm{mg}, n=126$ & $\begin{array}{l}\text { BIL } 10 \mathrm{mg} / \\
20 \mathrm{mg} \\
n=252 ; \\
\mathrm{PL} n=126\end{array}$ & 3 days & $A E$, sedative effects \\
\hline $\begin{array}{l}\text { Hindmarch [24], } \\
2002\end{array}$ & $\begin{array}{l}\text { RCT, } \\
\text { crossover } \\
\text { study }\end{array}$ & healthy & 15 & FEX $360 \mathrm{mg}, n=15$ & $\begin{array}{l}\text { PRO } 30 \mathrm{mg} n=15 \\
\mathrm{PL} n=15\end{array}$ & $7 \mathrm{~h}$ & $\begin{array}{l}\text { AE, sedative effects, CFF, } \\
\text { CRT, LARS }\end{array}$ \\
\hline $\begin{array}{l}\text { Hindmarch [15], } \\
1999\end{array}$ & $\begin{array}{l}\text { RCT, } \\
\text { crossover } \\
\text { study }\end{array}$ & healthy & 24 & FEX $120 \mathrm{mg}, n=24$ & $\begin{array}{l}\text { PRO } 30 \mathrm{mg} \\
n=24 ; \\
\text { LOR } 10 \mathrm{mg} \\
n=24 ; \\
\mathrm{PL} n=24\end{array}$ & $24 \mathrm{~h}$ & $\begin{array}{l}\text { AE, sedative effects, CFF, } \\
\text { CRT, LARS }\end{array}$ \\
\hline Horak [20], 2001 & $\begin{array}{l}\text { RCT, } \\
\text { crossover } \\
\text { study }\end{array}$ & $A R$ & 40 & FEX $120 \mathrm{mg}, n=40$ & $\begin{array}{l}\text { CET } 10 \mathrm{mg} \\
n=40 \\
\text { PL } n=40\end{array}$ & 2 days & $A E$, sedative effects \\
\hline Horak [48], 2010 & $\begin{array}{l}\text { RCT, } \\
\text { crossover } \\
\text { study }\end{array}$ & $\begin{array}{l}\text { allergic } \\
\text { volunteers }\end{array}$ & 75 & FEX $120 \mathrm{mg}, n=70$ & $\begin{array}{l}\text { CET } 10 \mathrm{mg} \\
n=68 ; \\
\text { BIL } 20 \mathrm{mg} \\
n=74 ; \\
\mathrm{PL} n=70\end{array}$ & 2 days & $A E$, sedative effects \\
\hline Horak [34], 2005 & $\begin{array}{l}\text { RCT, } \\
\text { crossover } \\
\text { study }\end{array}$ & $\begin{array}{l}\text { allergic } \\
\text { volunteers }\end{array}$ & 94 & FEX $120 \mathrm{mg}, n=94$ & $\mathrm{PL} n=94$ & 2 days & $A E$, sedative effects \\
\hline Howarth [16], 1999 & $\begin{array}{l}\mathrm{RCT} \text {, parallel } \\
\text { study }\end{array}$ & $A R$ & 722 & $\begin{array}{l}\text { FEX } 120 \mathrm{mg} / 180 \mathrm{mg}, \\
n=421\end{array}$ & $\begin{array}{l}\text { CET } 10 \mathrm{mg} \\
n=209 \\
\text { PL } n=209\end{array}$ & 2 weeks & $A E$, sedative effects \\
\hline Inami [53], 2016 & $\begin{array}{l}\text { RCT, } \\
\text { crossover } \\
\text { study }\end{array}$ & healthy & 20 & $\begin{array}{l}\text { FEX } 60 \mathrm{mg} \\
n=20\end{array}$ & $\begin{array}{l}\text { DIP } 50 \mathrm{mg} \\
n=20 \\
\text { PL } n=20\end{array}$ & $6 \mathrm{~h}$ & $A E$, sedative effects, LARS \\
\hline Kaiser [47], 2008 & $\begin{array}{l}\mathrm{RCT} \text {, parallel } \\
\text { study }\end{array}$ & $A R$ & 835 & FEX $120 \mathrm{mg}, n=359$ & $\begin{array}{l}\text { LOR } 10 \mathrm{mg} \\
n=357 \\
\text { PL } n=119\end{array}$ & 7 days & $A E$, sedative effects \\
\hline Kaiser [21], 2001 & $\begin{array}{l}\mathrm{RCT} \text {, parallel } \\
\text { study }\end{array}$ & $A R$ & 836 & FEX $120 \mathrm{mg}, n=360$ & $\begin{array}{l}\text { LOR } 10 \mathrm{mg} \\
n=357 \\
\text { PL } n=119\end{array}$ & 7 days & $A E$, sedative effects \\
\hline Kamei [49], 2012 & $\begin{array}{l}\text { RCT, } \\
\text { crossover } \\
\text { study }\end{array}$ & healthy & 24 & $\begin{array}{l}\text { FEX } 60 \mathrm{mg} \\
n=24\end{array}$ & $\begin{array}{l}\text { PRO } 25 \text { mg, } \\
n=24 ; \\
\text { OLO } 5 \mathrm{mg} \\
n=24 \\
\mathrm{PL} n=24\end{array}$ & $8 \mathrm{~h}$ & $\begin{array}{l}\text { AE, sedative effects, CFF, } \\
\text { CRT, LARS }\end{array}$ \\
\hline Kamei [28], 2003 & $\begin{array}{l}\text { RCT, } \\
\text { crossover } \\
\text { study }\end{array}$ & healthy & 11 & FEX $120 \mathrm{mg}, n=11$ & $\begin{array}{l}\mathrm{d}-\mathrm{CHL} 4 \mathrm{mg} \\
n=11 \\
\text { OLO } 10 \mathrm{mg} \\
n=11 \\
\mathrm{PL} n=11\end{array}$ & $8 \mathrm{~h}$ & $\begin{array}{l}\text { AE, sedative effects, CFF, } \\
\text { CRT, CTT, LARS }\end{array}$ \\
\hline $\begin{array}{l}\text { Mansfield [29], } \\
2003\end{array}$ & $\begin{array}{l}\text { RCT, } \\
\text { crossover } \\
\text { study }\end{array}$ & healthy & 42 & FEX $180 \mathrm{mg}, n=42$ & $\begin{array}{l}\text { DIP } 50 m g \\
n=42 \\
P L=42\end{array}$ & $2 \mathrm{~h}$ & $A E$, sedative effects \\
\hline Milgrom [46], 2007 & $\begin{array}{l}\text { RCT, parallel } \\
\text { study }\end{array}$ & $A R$ & 453 & $\begin{array}{l}\text { FEX } 60 \mathrm{mg} \\
n=222\end{array}$ & $P L n=231$ & 2 weeks & $A E$, sedative effects \\
\hline Okubo [44], 2006 & $\begin{array}{l}\text { RCT, } \\
\text { crossover } \\
\text { study }\end{array}$ & healthy & 9 & FEX $60 \mathrm{mg}, n=9$ & $\begin{array}{l}\text { EPI } 20 m g \\
n=9 \\
P L n=9\end{array}$ & $5 \mathrm{~h}$ & $A E$, sedative effects \\
\hline
\end{tabular}


Table 1 Characteristics of included studies (Continued)

\begin{tabular}{|c|c|c|c|c|c|c|c|}
\hline First author, Years & Study design & Subject & Number & Experimental & Comparators & $\begin{array}{l}\text { Study } \\
\text { duration }\end{array}$ & Outcome measures \\
\hline Okubo [54], 2016 & $\begin{array}{l}\text { RCT, parallel } \\
\text { study }\end{array}$ & $A R$ & 747 & FEX $120 \mathrm{mg}, n=247$ & $\begin{array}{l}\text { BIL } 20 \mathrm{mg} \\
n=249 \\
\text { PL } n=251\end{array}$ & 2 weeks & $A E$, sedative effects \\
\hline Prenner [18], 2000 & $\begin{array}{l}\text { RCT, } \\
\text { crossover } \\
\text { study }\end{array}$ & AR & 929 & FEX $120 \mathrm{mg}, n=457$ & $\begin{array}{l}\text { LOR } 10 \mathrm{mg} \\
n=472\end{array}$ & 14 days & $A E$, sedative effects \\
\hline Purohit [22], 2001 & $\begin{array}{l}\text { RCT, } \\
\text { crossover } \\
\text { study }\end{array}$ & healthy & 26 & $\begin{array}{l}\text { FEX } 120 \mathrm{mg} / 180 \mathrm{mg} \\
n=52\end{array}$ & $\begin{array}{l}\text { CET } 10 \text { mg, } \\
n=26 ; \\
\text { PL } n=26\end{array}$ & $24 \mathrm{~h}$ & $A E$, sedative effects \\
\hline Purohit [35], 2004 & $\begin{array}{l}\text { RCT, } \\
\text { crossover } \\
\text { study }\end{array}$ & healthy & 42 & FEX $180 \mathrm{mg}, \mathrm{n}=42$ & $\begin{array}{l}\text { CET } 10 \mathrm{mg}, \\
n=42\end{array}$ & $4 \mathrm{~h}$ & $A E$, sedative effects \\
\hline Ridout [30], 2003 & $\begin{array}{l}\text { RCT, } \\
\text { crossover } \\
\text { study }\end{array}$ & healthy & 18 & FEX $180 \mathrm{mg}, n=18$ & $\begin{array}{l}\text { HYD } 50 \text { mg, } \\
n=18 \\
\text { PL } n=18\end{array}$ & $5 \mathrm{~h}$ & $\begin{array}{l}\text { AE, sedative effects, CFF, } \\
\text { CRT, LARS }\end{array}$ \\
\hline $\begin{array}{l}\text { Schapowal [39], } \\
2005\end{array}$ & $\begin{array}{l}\text { RCT, parallel } \\
\text { study }\end{array}$ & $A R$ & 220 & FEX $180 \mathrm{mg}, n=113$ & $\mathrm{PL} n=107$ & 14 days & $A E$, sedative effects \\
\hline $\begin{array}{l}\text { Schoepke [51], } \\
2013\end{array}$ & $\begin{array}{l}\text { RCT, } \\
\text { crossover } \\
\text { study }\end{array}$ & healthy & 18 & FEX $120 \mathrm{mg}, n=18$ & $\mathrm{PL} n=18$ & $24 \mathrm{~h}$ & $A E$, sedative effects \\
\hline Simons [9], 1997 & $\begin{array}{l}\text { RCT, } \\
\text { crossover } \\
\text { study }\end{array}$ & healthy & 20 & FEX $120 \mathrm{mg}, n=40$ & $\begin{array}{l}\text { LOR } 10 \mathrm{mg} \\
n=20 \\
\mathrm{PL} n=20\end{array}$ & $24 \mathrm{~h}$ & $A E$, sedative effects \\
\hline Tsuda [40], 2005 & $\begin{array}{l}\text { RCT, } \\
\text { crossover } \\
\text { study }\end{array}$ & healthy & 10 & FEX $120 \mathrm{mg}, n=10$ & $\begin{array}{l}\text { CET } 5 \mathrm{mg} / 10 \mathrm{mg} \\
n=20 ; \\
\text { LOR } 10 \mathrm{mg} \\
n=10 \\
\text { PL } n=10\end{array}$ & $24 \mathrm{~h}$ & $A E$, sedative effects \\
\hline Wahn [32], 2003 & $\begin{array}{l}\text { RCT, parallel } \\
\text { study }\end{array}$ & $A R$ & 935 & $\begin{array}{l}\text { FEX } 30 \mathrm{mg} \\
n=464\end{array}$ & $\mathrm{PL} n=471$ & 14 days & $A E$, sedative effects \\
\hline Weiler [19], 2000 & $\begin{array}{l}\text { RCT, } \\
\text { crossover } \\
\text { study }\end{array}$ & healthy & 40 & $\begin{array}{l}\text { FEX } 60 \mathrm{mg} \\
n=40\end{array}$ & $\begin{array}{l}\text { DIP } 50 \text { mg, } \\
n=40 \\
P L=40\end{array}$ & $5 \mathrm{~h}$ & $\begin{array}{l}\text { AE, sedative effects, VAS of } \\
\text { drowsiness }\end{array}$ \\
\hline $\begin{array}{l}\text { Ballmer-Weber [12], } \\
1999\end{array}$ & $\begin{array}{l}\text { RCT, } \\
\text { crossover } \\
\text { study }\end{array}$ & healthy & 20 & FEX $180 \mathrm{mg}, n=20$ & $\begin{array}{l}\text { CET } 10 \mathrm{mg} \\
n=40 \\
\text { ACR } 8 \text { mg } \\
n=20\end{array}$ & $1 \mathrm{~h}$ & sedative effects \\
\hline Day [37], 2005 & $\begin{array}{l}\text { RCT, parallel } \\
\text { study }\end{array}$ & AR & 599 & FEX $180 \mathrm{mg}, n=250$ & $\begin{array}{l}\text { CET } 10 \text { mg, } \\
n=249 \\
\text { PL } n=100\end{array}$ & $7 \mathrm{~h}$ & sedative effects \\
\hline Handa [27], 2004 & $\begin{array}{l}\text { RCT, parallel } \\
\text { study }\end{array}$ & CIU & 97 & FEX $180 \mathrm{mg}, n=45$ & $\begin{array}{l}\text { CET } 10 \mathrm{mg} \\
n=52\end{array}$ & 28 days & sedative effects \\
\hline Hyo [38], 2005 & $\begin{array}{l}\text { RCT, parallel } \\
\text { study }\end{array}$ & healthy & 113 & FEX $120 \mathrm{mg}, n=28$ & $\begin{array}{l}\text { CET } 10 \text { mg; } \\
n=30 ; \\
\text { LOR } 10 \mathrm{mg} \\
n=28 \\
\text { PL } n=27\end{array}$ & 2 days & sedative effects \\
\hline Ridout [58], 2002 & $\begin{array}{l}\text { RCT, } \\
\text { crossover } \\
\text { study }\end{array}$ & healthy & 24 & FEX $120 \mathrm{mg}, n=24$ & $\begin{array}{l}\text { PRO } 25 \mathrm{mg} \\
n=24 ; \\
\text { PL } n=24\end{array}$ & $8 \mathrm{~h}$ & CFF, CRT, CTT, LARS \\
\hline Naicker [59], 2013 & $\begin{array}{l}\text { RCT, } \\
\text { crossover } \\
\text { study }\end{array}$ & healthy & 11 & FEX $180 \mathrm{mg}, n=11$ & $\begin{array}{l}\text { PRO } 25 \mathrm{mg}, n=11 ; \\
\text { LOR } 10 \mathrm{mg} \\
n=11 ; \\
\mathrm{PL} n=11\end{array}$ & $3 \mathrm{~h}$ & $\begin{array}{l}\text { CRT, CTT, VAS of } \\
\text { drowsiness }\end{array}$ \\
\hline
\end{tabular}




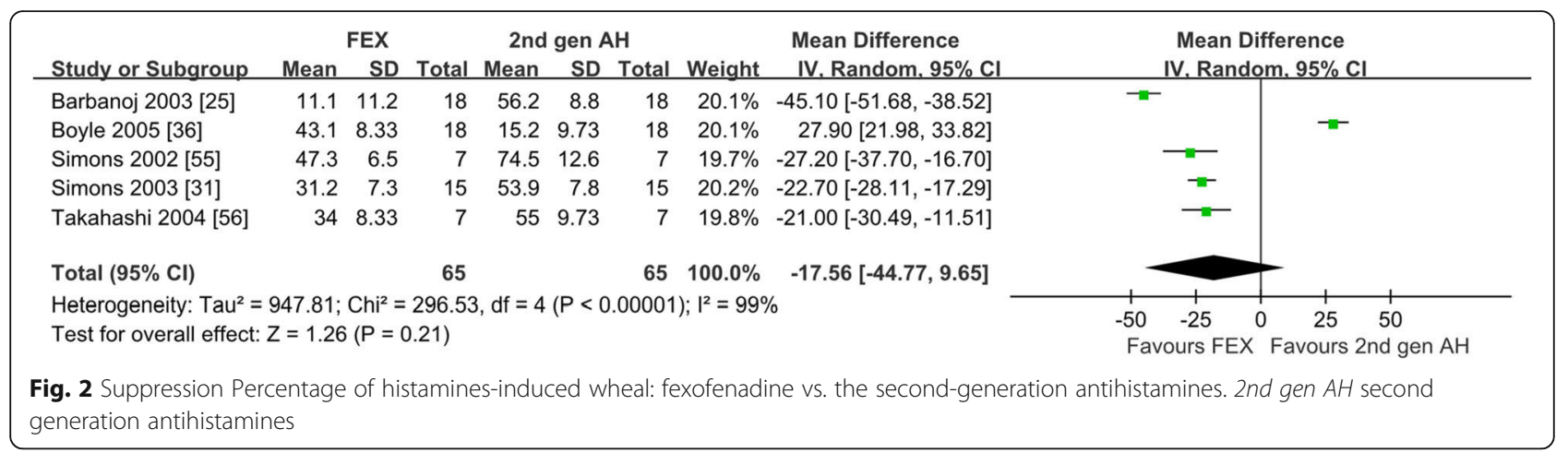

\section{Safety}

\section{Adverse events frequency}

Forty-one studies reported AE after taking fexofenadine [9-11, 13-26, 28-30, 32-36, 39-54]. Of 41 studies, 10 compared with the first-generation antihistamines [15, 19, 24, 28-30, 43, 49, 50, 53], 22 compared with the second-generation antihistamines $[9,14,16-18,20-23$, $25,28,33,35,36,40,44,47-49,52-54]$, and 37 compared with placebo $[9-11,13-17,19-25,28-30,32-34$, 36, 39-49, 51-54]. When compared with the firstgeneration antihistamines, as demonstrated in Fig. 4a, fexofenadine produced significantly lower $\mathrm{AE}$ frequency $(\mathrm{OR}=0.446 ; 95 \% \mathrm{CI}: 0.214$ to $0.929, P=0.031)$. When compared with the second-generation antihistamines, as shown in Fig. 4b, the AE frequency for fexofenadine versus the second-generation antihistamines were not different $(\mathrm{OR}=0.987 ; 95 \% \mathrm{CI}: 0.815$ to $1.195, P=0.890)$. When compared with placebo, as shown in Additional file 4: Figure $\mathrm{S} 4$, the AE frequency of these two groups were not different $(\mathrm{OR}=0.999 ; 95 \% \mathrm{CI}$ : 0.863 to 1.156 , $P=0.987)$.

\section{Sedative effects frequency}

Forty-six studies reported sedative effects frequency after taking fexofenadine [9-54]. Of 46 studies, 10 compared with the first-generation antihistamines $[15,19,24,28-$ $30,43,49,50,53], 27$ compared with the secondgeneration antihistamines $[9,12,14,16-18,20-23,25$,
27, 28, 31, 33, 35-38, 40, 44, 47-49, 52-54], and 38 compared with placebo $[9-11,13-17,19-25,28-30$, $32-34,36,38-49,51-54]$. When compared with the first-generation antihistamines, as shown in Fig. 5a, fexofenadine produced significantly lower sedative effects frequency $(\mathrm{OR}=0.265$; $95 \% \mathrm{CI}$ : 0.072 to $0.976, P=$ 0.046). When compared with the second-generation antihistamines, as shown in Fig. 5b, fexofenadine produced significantly lower sedative effects frequency $(\mathrm{OR}=$ 0.578; 95\% CI: 0.369 to $0.906, P=0.017)$. When compared with placebo, as shown in Additional file 5: Figure $\mathrm{S} 5$, the sedative effects frequency for fexofenadine versus placebo were not different $(\mathrm{OR}=1.608 ; 95 \% \mathrm{CI}$ : 0.884 to $2.924, P=0.120$ ), but not statistically significant (OR 1.6 [0.8-2.9]). Five studies $(18,432,126,113,747$ patients respectively) showed more AE for fexofenadine than placebo and none of the others showed that for placebo more AEs than fexofenadine.

\section{Cognitive/psychomotor function}

CFF. Six studies reported the CFF of subjects after taking fexofenadine $[15,24,28,30,49,58]$. Of the 6 studies on healthy subjects, 3 compared with the secondgeneration antihistamines [15, 28, 49], 6 compared with the first-generation antihistamines and placebo [15, 24, $28,30,49,58]$. When compared with the firstgeneration antihistamines, as shown in Fig. 6a, fexofenadine produced significantly less change of CFF (WMD =

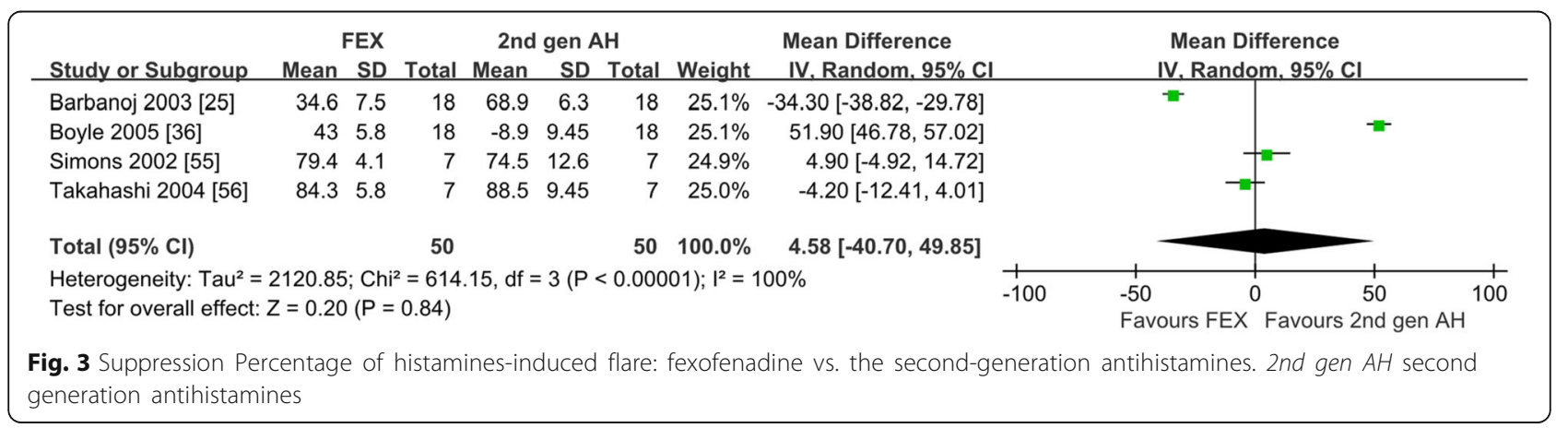


a

Frequency of adverse events: fexofenadine vs. the first-generation antihistamines

\begin{tabular}{|c|c|c|c|c|c|}
\hline \multirow[t]{2}{*}{$\underline{\text { Study name }}$} & \multicolumn{5}{|c|}{ Statistics for each study } \\
\hline & $\begin{array}{c}\text { Peto } \\
\text { odds ratio }\end{array}$ & $\begin{array}{c}\text { Lower } \\
\text { limit }\end{array}$ & $\begin{array}{l}\text { Upper } \\
\text { limit }\end{array}$ & Z-Value & p-Value \\
\hline Boyle 2006 [43] & 2.028 & 0.197 & 20.860 & 0.595 & 0.552 \\
\hline Hindmarch 1999 [15] & 0.456 & 0.160 & 1.300 & -1.469 & 0.142 \\
\hline Hindmarch 2002 [24] & 1.000 & 0.000 & 46757334002463.398 & 0.000 & 1.000 \\
\hline Inami 2016 [53] & 1.000 & 0.000 & 775095803516.189 & 0.000 & 1.000 \\
\hline Kamei 2003 [28] & 1.000 & 0.000 & 7286995580067600.000 & 0.000 & 1.000 \\
\hline Kamei 2012 [49] & 1.000 & 0.000 & 75268506707.877 & 0.000 & 1.000 \\
\hline Mansfield 2012 [29] & 1.000 & 0.000 & 181697035.105 & 0.000 & 1.000 \\
\hline Ramesh 2013 [50] & 0.294 & 0.092 & 0.938 & -2.069 & 0.039 \\
\hline Ridout 2003 [30] & 1.000 & 0.000 & 3270511690688.450 & 0.000 & 1.000 \\
\hline Weiler 2000 [19] & 1.000 & 0.000 & 288910611.492 & 0.000 & 1.000 \\
\hline & 0.446 & 0.214 & 0.929 & -2.156 & 0.031 \\
\hline
\end{tabular}

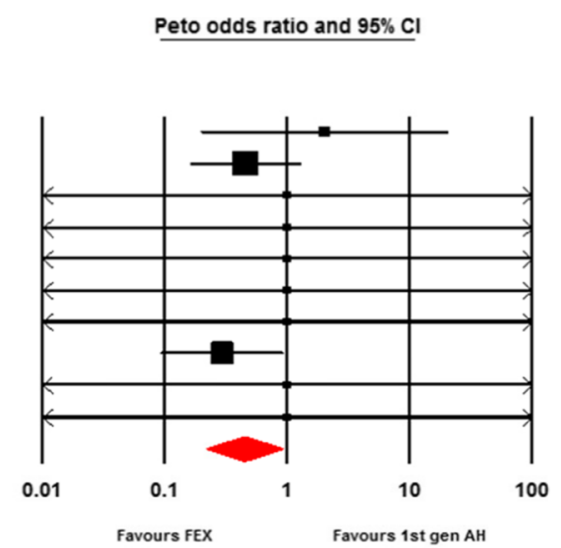

b

Frequency of adverse events: fexofenadine vs. the second-generation antihistamines

$\begin{array}{lrc}\text { Study name } & \begin{array}{c}\text { Peto } \\ \text { odds ratio }\end{array} & \begin{array}{c}\text { Lower } \\ \text { limit }\end{array} \\ \text { Barbanoj 2003 [25] } & 1.000 & 0.274 \\ \text { Boyle 2005 [36] } & 1.000 & 0.000 \\ \text { Day 2004 [33] } & 1.422 & 0.869 \\ \text { Grant 1999 [14] } & 1.000 & 0.000 \\ \text { Grant 2002 [23] } & 2.830 & 0.473 \\ \text { Hashiguchi 2016 [52] } & 0.884 & 0.380 \\ \text { Horak 2001 [20] } & 1.978 & 0.200 \\ \text { Horak 2010 [48] } & 0.220 & 0.027 \\ \text { Howarth 1999 [16] } & 0.903 & 0.612 \\ \text { Inami 2016 [53] } & 1.000 & 0.000 \\ \text { Kaiser 2001 [21] } & 0.741 & 0.437 \\ \text { Kaiser 2008 [47] } & 0.743 & 0.438 \\ \text { Kamei 2003 [28] } & 1.000 & 0.000 \\ \text { Kamei 2012 [49] } & 1.000 & 0.000 \\ \text { Okubo 2006 [44] } & 1.000 & 0.000 \\ \text { Okubo 2016 [54] } & 0.730 & 0.360 \\ \text { Prenner 2000 [18] } & 1.611 & 0.966 \\ \text { Purohit 2001 [22] } & 1.000 & 0.000 \\ \text { Purohit 2004 [35] } & 0.132 & 0.008 \\ \text { Simons 1997 [9] } & 1.000 & 0.000 \\ \text { Tsuda 2005 [40] } & 1.000 & 0.000 \\ \text { Van Cauwenberge 2000 [17] } & 1.183 & 0.358 \\ & 0.987 & 0.815\end{array}$

\begin{tabular}{|c|c|c|}
\hline \multicolumn{3}{|l|}{$\underline{\text { Statistics for each study }}$} \\
\hline $\begin{array}{c}\text { Upper } \\
\text { limit }\end{array}$ & Z-Value & p-Value \\
\hline 3.656 & 0.000 & 1.000 \\
\hline 3270511690688.450 & 0.000 & 1.000 \\
\hline 2.326 & 1.401 & 0.161 \\
\hline 120525219998.684 & -0.000 & 1.000 \\
\hline 16.917 & 1.140 & 0.254 \\
\hline 2.055 & -0.287 & 0.774 \\
\hline 19.512 & 0.584 & 0.559 \\
\hline 1.794 & -1.414 & 0.157 \\
\hline 1.334 & -0.511 & 0.609 \\
\hline 775095803516.189 & 0.000 & 1.000 \\
\hline 1.257 & -1.112 & 0.266 \\
\hline 1.261 & -1.101 & 0.271 \\
\hline 7286995580067600.000 & 0.000 & 1.000 \\
\hline 75268506707.877 & 0.000 & 1.000 \\
\hline 277762504064972000.000 & 0.000 & 1.000 \\
\hline 1.477 & -0.876 & 0.381 \\
\hline 2.687 & 1.828 & 0.067 \\
\hline 28738230973.536 & 0.000 & 1.000 \\
\hline 2.147 & -1.423 & 0.155 \\
\hline 21897384494.852 & 0.000 & 1.000 \\
\hline 53535894653355.797 & 0.000 & 1.000 \\
\hline 3.908 & 0.276 & 0.783 \\
\hline 1.195 & -0.138 & 0.890 \\
\hline
\end{tabular}

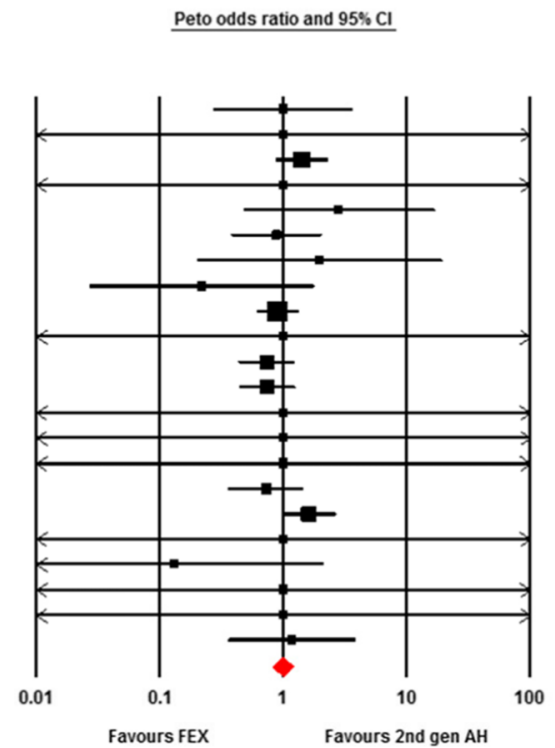

Fig. 4 Frequency of adverse events: a fexofenadine vs. the first-generation antihistamines; $\mathbf{b}$ fexofenadine vs. the second-generation antihistamines. 1st gen AH the first-generation antihistamines, 2nd gen AH second generation antihistamines

1.73; $95 \%$ CI: 1.14 to $2.32, P<0.00001)$ and the subgroup-analysis showed that fexofenadine $120 \mathrm{mg} / \mathrm{d}$ produced significantly less change of CFF compared with promethazine (WMD $=1.62$; $95 \% \mathrm{CI}: 1.33$ to $1.91, P<$ $0.00001)$. When compared with the second-generation antihistamines, as shown in Fig. 6b, the change of CFF were not different (WMD $=0.20 ; 95 \% \mathrm{CI}$ : -0.16 to 0.56 , $P=0.28$ ) and the subgroup-analysis showed that fexofenadine produced significantly less change of CFF compared with olopatadine (WMD $=0.37$; $95 \% \mathrm{CI}$ : 0.24 to $0.49, P<0.00001)$. When compared with placebo, as shown in Additional file 6: Figure S6, the change of CFF were not different $(\mathrm{WMD}=-0.15 ; 95 \% \mathrm{CI}:-0.37$ to
0.06, $P=0.17$ ). After checking the trial methods, we found that the heterogeneity for fexofenadine versus placebo may be generated from the inconsistent doses of fexofenadine. Sensitivity analysis showed the metaanalysis results were similar.

CRT. Seven studies reported the CRT of subjects after taking fexofenadine $[15,24,28,30,49,58,59]$. Of the 7 studies on healthy subjects, all of the included studies compared with the first-generation antihistamines [15, 24, $28,30,49,58,59]$, as shown in Fig. 7a, fexofenadine produced significantly less change of CRT (WMD $=-61.41$; 95\% CI: -81.87 to $-40.96, P<0.00001)$. Four studies compared with the second-generation antihistamines $[15$, 


\begin{tabular}{|c|c|c|c|c|c|}
\hline & & requenc & of sedative effects: fexc & fenadine & s. the $f i$ \\
\hline \multirow[t]{2}{*}{ Study name } & \multicolumn{5}{|c|}{ Statistics for each study } \\
\hline & $\begin{array}{c}\text { Peto } \\
\text { odds ratio }\end{array}$ & $\begin{array}{c}\text { Lower } \\
\text { limit }\end{array}$ & $\begin{array}{l}\text { Upper } \\
\text { limit }\end{array}$ & $z$-Value & $\mathrm{p}-\mathrm{Va}_{\mathrm{a}}$ \\
\hline Boyle 2006 [43] & 1.000 & 0.060 & 16.648 & 0.000 & \\
\hline Hindmarch 1999 [15] & 1.000 & 0.000 & 846896741.958 & 0.000 & 1.0 \\
\hline Hindmarch 2002 [24] & 1.000 & 0.000 & 46757334002463.398 & 0.000 & 1.0 \\
\hline Inami 2016 [53] & 1.000 & 0.000 & 775095803516.189 & 0.000 & 1.0 \\
\hline Kamei 2003 [28] & 1.000 & 0.000 & 7286995580067600.000 & 0.000 & \\
\hline Kamei 2012 [49] & 1.000 & 0.000 & 75268506707.877 & 0.000 & \\
\hline Mansfield 2003 [29] & 1.000 & 0.000 & 181697035.105 & 0.000 & \\
\hline Ramesh 2013 [50] & 0.174 & 0.039 & 0.776 & -2.291 & \\
\hline Ridout 2003 [30] & 1.000 & 0.000 & 156845750.313 & 0.000 & \\
\hline \multirow[t]{2}{*}{ Weiler 2000 [19] } & 1.000 & 0.000 & 288910611.492 & 0.000 & \\
\hline & 0.265 & 0.072 & 0.976 & -1.996 & 0.0 \\
\hline
\end{tabular}

b

Frequency of sedative effects: fexofenadine vs. the second-generation antihistamines

\begin{tabular}{|c|c|c|c|c|c|}
\hline \multirow[t]{2}{*}{ Study name } & \multicolumn{5}{|c|}{$\underline{\text { Statistics for each study }}$} \\
\hline & $\begin{array}{c}\text { Peto } \\
\text { odds ratio }\end{array}$ & $\begin{array}{c}\text { Lower } \\
\text { limit }\end{array}$ & $\begin{array}{l}\text { Upper } \\
\text { Imit }\end{array}$ & Z-Value & p-Value \\
\hline Ballmer-Webber 1999 [12] & 0.356 & 0.087 & 1.456 & -1.437 & 0.151 \\
\hline Barbanoj 2003 [25] & 1.264 & 0.331 & 4.832 & 0.342 & 0.732 \\
\hline Boyle 2005 [36] & 1.000 & 0.000 & 3270511690688.450 & 0.000 & 1.000 \\
\hline Day 2004 [33] & 1.000 & 0.000 & 2991.471 & 0.000 & 1.000 \\
\hline Day 2005 [37] & 0.134 & 0.008 & 2.152 & -1.418 & 0.156 \\
\hline Grant 1999 [14] & 1.000 & 0.000 & 120525219998.684 & -0.000 & 1.000 \\
\hline Grant $2002[23]$ & 1.000 & 0.099 & 10.100 & 0.000 & 1.000 \\
\hline Handa 2003 [27] & 0.575 & 0.111 & 2.986 & -0.659 & 0.510 \\
\hline Hashiguchi 2016 [52] & 20.086 & 0.314 & 1283.969 & 1.414 & 0.157 \\
\hline Horak 2001 [20] & 1.000 & 0.000 & 96048806.473 & 0.000 & 1.000 \\
\hline Horak 2010 [48] & 1.000 & 0.000 & 352846.577 & 0.000 & 1.000 \\
\hline Howarth 1999 [16] & 0.419 & 0.203 & 0.863 & -2.358 & 0.018 \\
\hline Hyo 2005 [38] & 1.038 & 0.179 & 6.035 & 0.042 & 0.967 \\
\hline Inami 2016 [53] & 1.000 & 0.000 & 775095803516.189 & 0.000 & 1.000 \\
\hline Kaiser 2001 [21] & 1.000 & 0.001 & 695.057 & 0.000 & 1.000 \\
\hline Kaiser 2008 [47] & 1.000 & 0.001 & 698.212 & 0.000 & 1.000 \\
\hline Kamei 2003 [28] & 1.000 & 0.000 & 7286995580067600.000 & 0.000 & 1.000 \\
\hline Kamei 2012 [49] & 1.000 & 0.000 & 75268506707.877 & 0.000 & 1.000 \\
\hline Okubo 2006 [44] & 1.000 & 0.000 & 277762504064972000.000 & 0.000 & 1.000 \\
\hline Okubo 2016 [54] & 0.516 & 0.053 & 4.985 & -0.572 & 0.568 \\
\hline Prenner 2000 [18] & 1.000 & 0.003 & 314.393 & 0.000 & 1.000 \\
\hline Purohit 2001 [22] & 1.000 & 0.000 & 28738230973.536 & 0.000 & 1.000 \\
\hline Purohit 2004 [35] & 0.132 & 0.008 & 2.147 & -1.423 & 0.155 \\
\hline Simons 1997 [9] & 1.000 & 0.000 & 775095803516.189 & 0.000 & 1.000 \\
\hline Simons 2003 [31] & 1.000 & 0.238 & 4.205 & 0.000 & 1.000 \\
\hline Tsuda $2005[40]$ & 1.000 & 0.000 & 53535894653355.797 & 0.000 & 1.000 \\
\hline Van Cauwenberge 2000[17] & 1.000 & 0.000 & 3523.029 & 0.000 & 1.000 \\
\hline & 0.578 & 0.369 & 0.906 & -2.392 & 0.017 \\
\hline
\end{tabular}

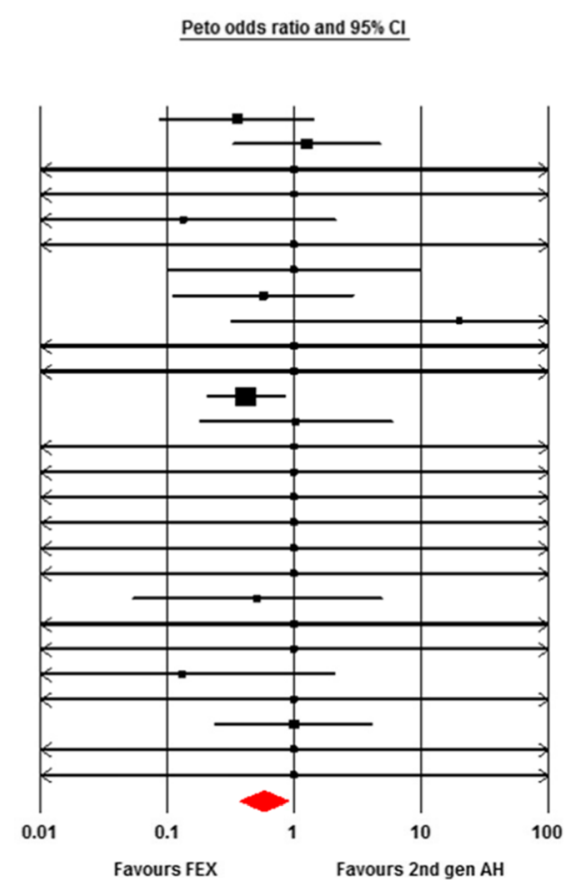

\begin{tabular}{|c|c|c|c|c|c|c|}
\hline Model & & \multicolumn{3}{|c|}{ Effect size and $95 \%$ interval } & \multicolumn{2}{|c|}{ Test of null (2-Tail) } \\
\hline Model & $\begin{array}{l}\text { Nunber } \\
\text { Studies }\end{array}$ & $\begin{array}{c}\text { Point } \\
\text { eslimate }\end{array}$ & $\begin{array}{c}\text { Lower } \\
\text { limi }\end{array}$ & $\begin{array}{l}\text { Upper } \\
\text { limk }\end{array}$ & Z-value & P-value \\
\hline Fixed & 27 & 0.578 & 0.369 & 0.906 & $\cdot 2.392$ & 0.017 \\
\hline Random & 27 & 0.578 & 0.369 & 0.906 & .2 .392 & 0.017 \\
\hline
\end{tabular}
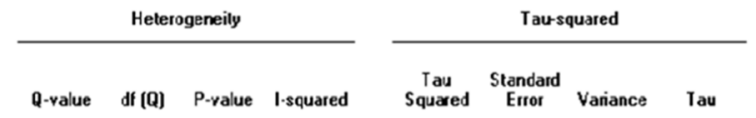

$0.030 \quad 0.439 \quad 0.220 \quad 0.000$

Fig. 5 Frequency of sedative effects: a fexofenadine vs. the first-generation antihistamines; $\mathbf{b}$ fexofenadine vs. the second-generation antihistamines. 1st gen $\mathrm{AH}$ the first-generation antihistamines, 2nd gen $\mathrm{AH}$ second generation antihistamines

$28,49,59]$, as shown in Fig. $7 \mathrm{~b}$, the change of CRT were not different (WMD $=5.28 ; 95 \% \mathrm{CI}:-3.07$ to 13.63, $P=0.22$ ). Six studies compared with placebo [24, 28, 30, 49, 58, 59], as shown in Additional file 7:
Figure S7, the change of CRT were not different (WMD $=3.68 ; 95 \% \mathrm{CI}:-2.95$ to $10.32, P=0.28$ ). The heterogeneity may be generated from the inconsistent doses of fexofenadine and different antihistamines in 


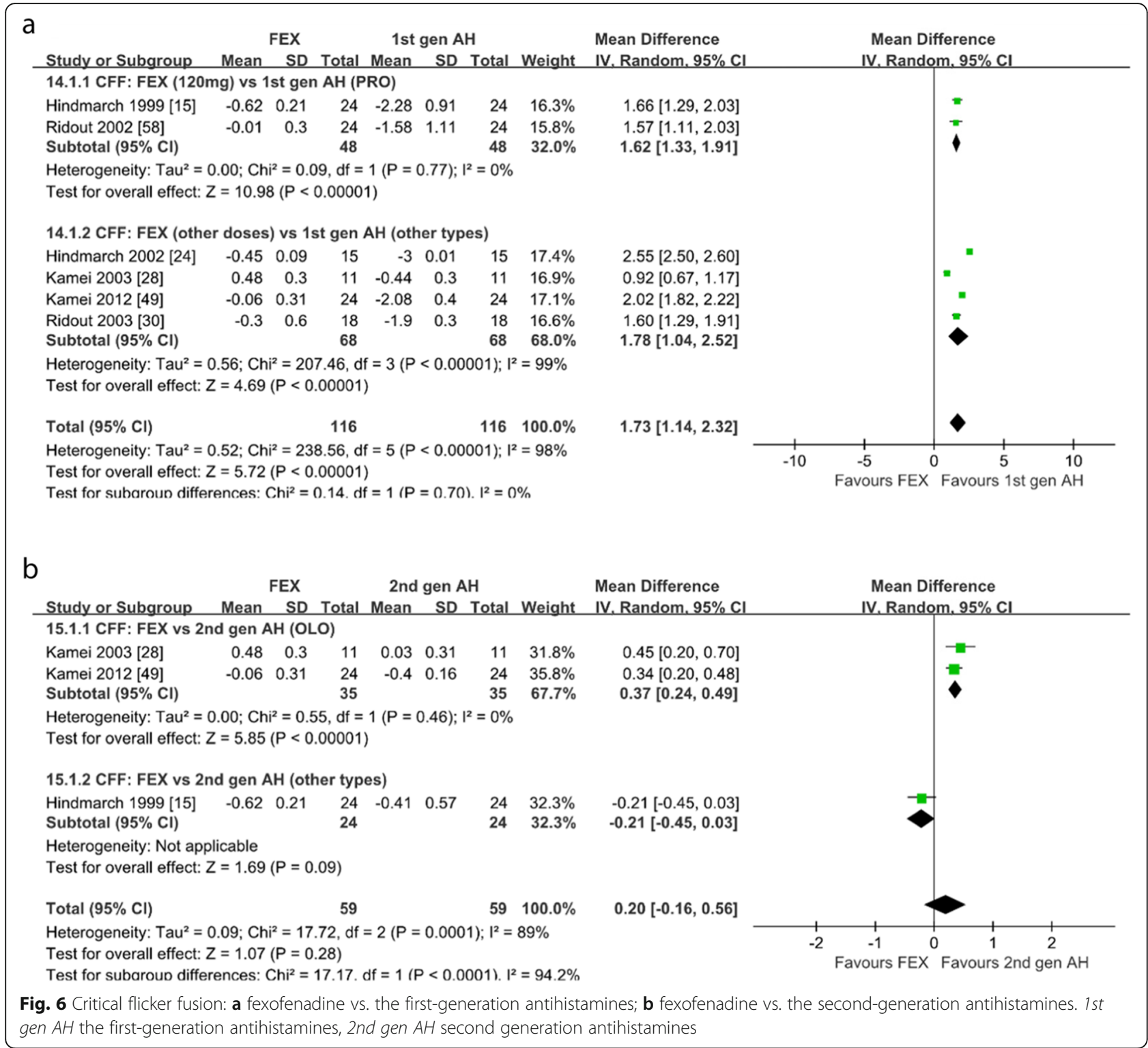

each comparator. Sensitivity analysis showed the meta-analysis results were similar.

CTT. Three studies on healthy subjects compared the CTT of subjects with the first-generation antihistamines, the second-generation antihistamines and placebo [28, $58,59]$. When compared with the first-generation antihistamines, as shown in Fig. 8a, fexofenadine produced significantly less change of CTT (WMD $=-21.79$; 95\% CI: -42.44 to $-1.14, P=0.04$ ) and the subgroup-analysis showed that fexofenadine $120 \mathrm{mg} / \mathrm{d}$ or less dose produced significantly less change of CTT (WMD = - 10.04; 95\% CI: -13.16 to $-6.44, P<0.00001)$. When compared with the second-generation antihistamines, as shown in Fig. 8b, fexofenadine produced significantly less change of CTT (WMD $=-2.43 ; 95 \% \mathrm{CI}:-3.67$ to $-1.18, P=$ 0.0001). When compared with placebo, as shown in
Additional file 8: Figure S8, the change of CTT were not different (WMD $=0.11$; 95\% CI: -3.81 to $4.02, P=0.96$ ). Sensitivity analysis showed the meta-analysis results were similar.

LARS. Seven studies reported the LARS of subjects after taking fexofenadine [15, 24, 28, 30, 49, 53, 58]. Of the 7 studies on healthy subjects, 3 compared with the second-generation antihistamines [15, 28, 49], all compared with the first-generation antihistamines and placebo $[15,24,28,30,49,53,58]$. As shown in Fig. 9 and Additional file 9: Figure S9, fexofenadine produced significantly less change of LARS when compared with the first-generation antihistamines $(\mathrm{WMD}=-6.34 ; 95 \% \mathrm{CI}$ : - 10.53 to $-2.15, P=0.003)$, the second-generation antihistamines (WMD $=-7.75$; 95\% CI: -12.56 to -2.93 , $P=0.002)$ and placebo $(\mathrm{WMD}=-2.67 ; 95 \% \mathrm{CI}:-3.99$ 


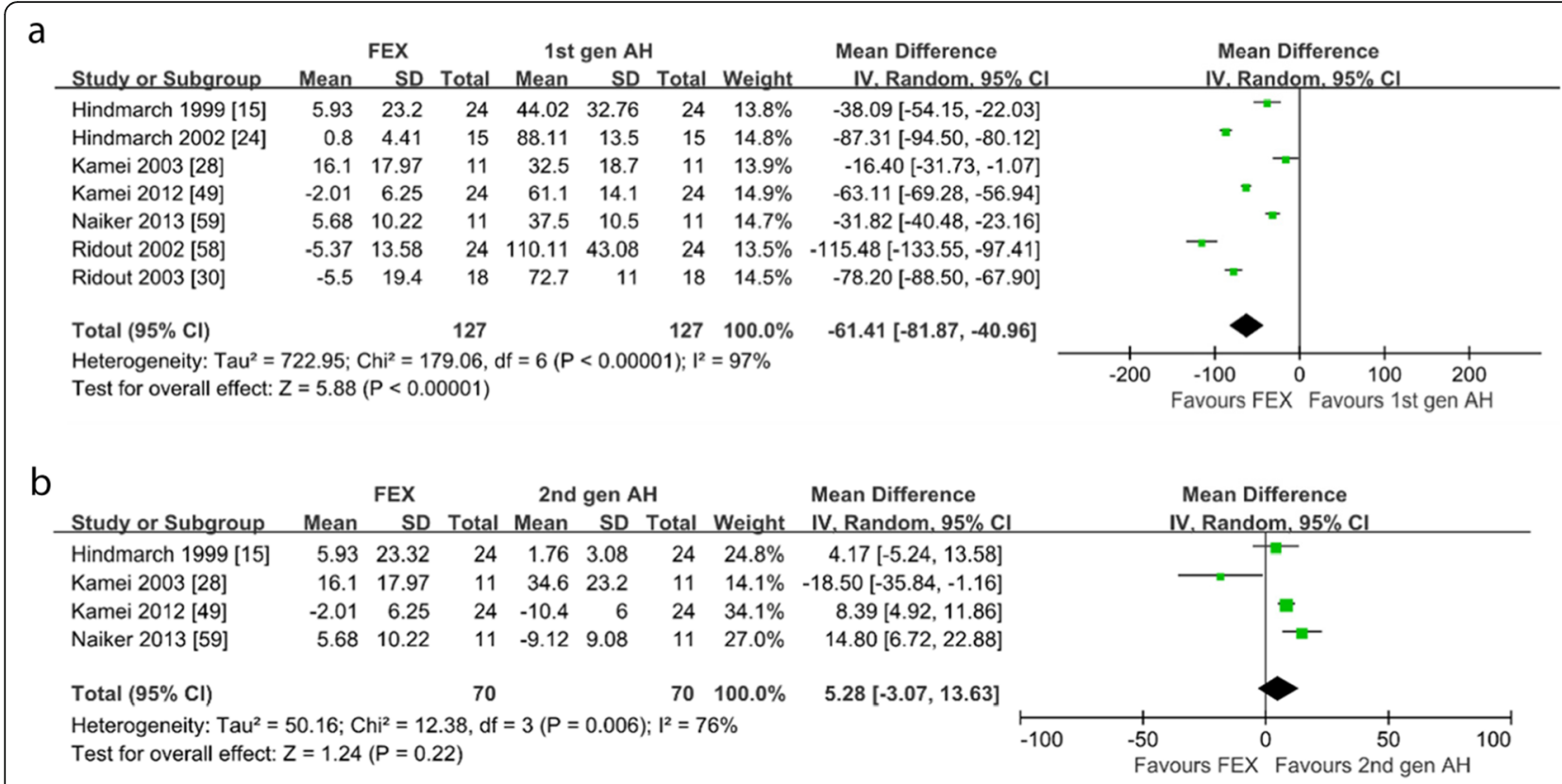

Fig. 7 Choice reaction time: a fexofenadine vs. the first-generation antihistamines; $\mathbf{b}$ fexofenadine vs. the second-generation antihistamines. 1st gen $A H$ the first-generation antihistamines, 2nd gen AH second generation antihistamines

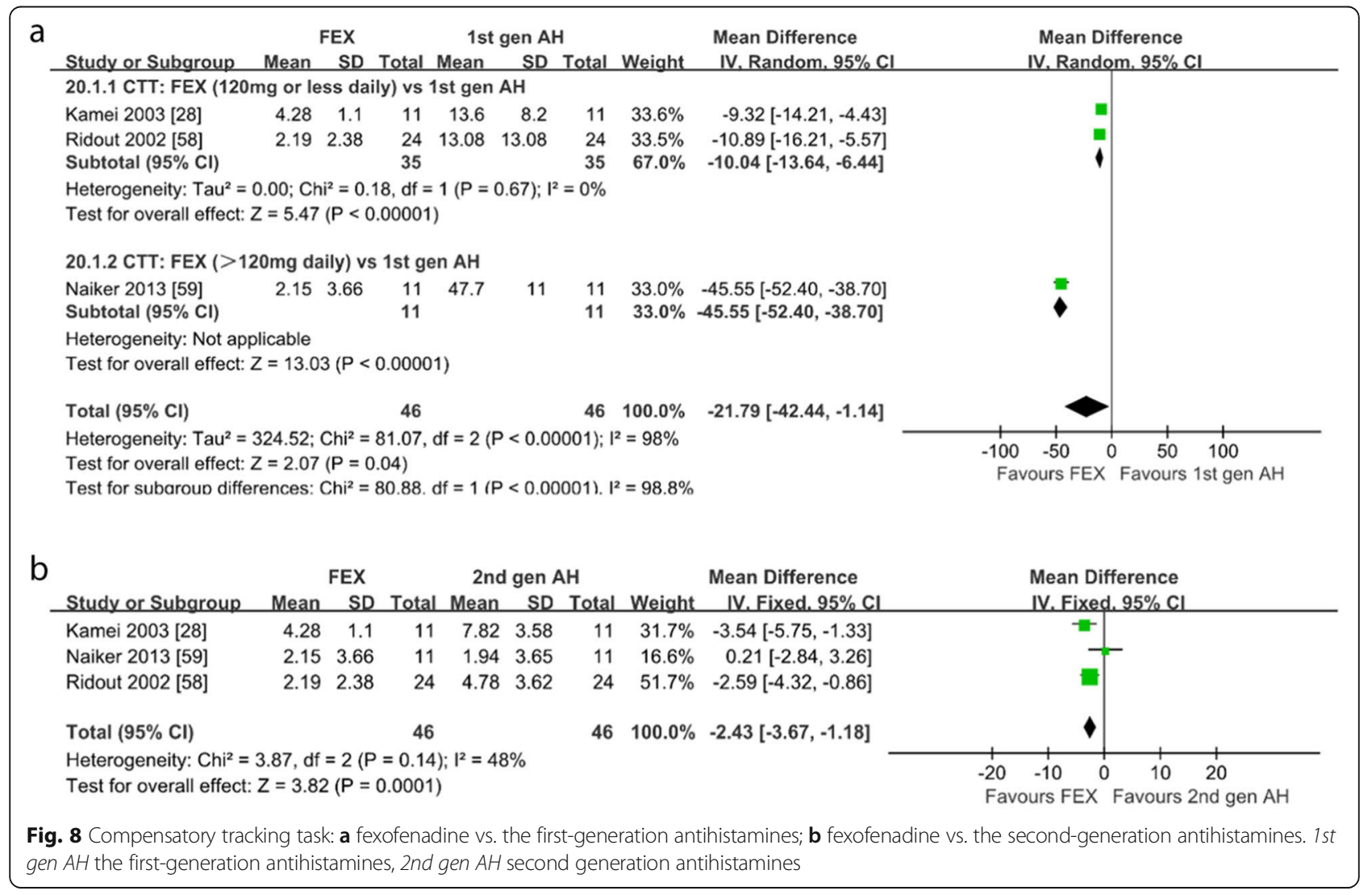




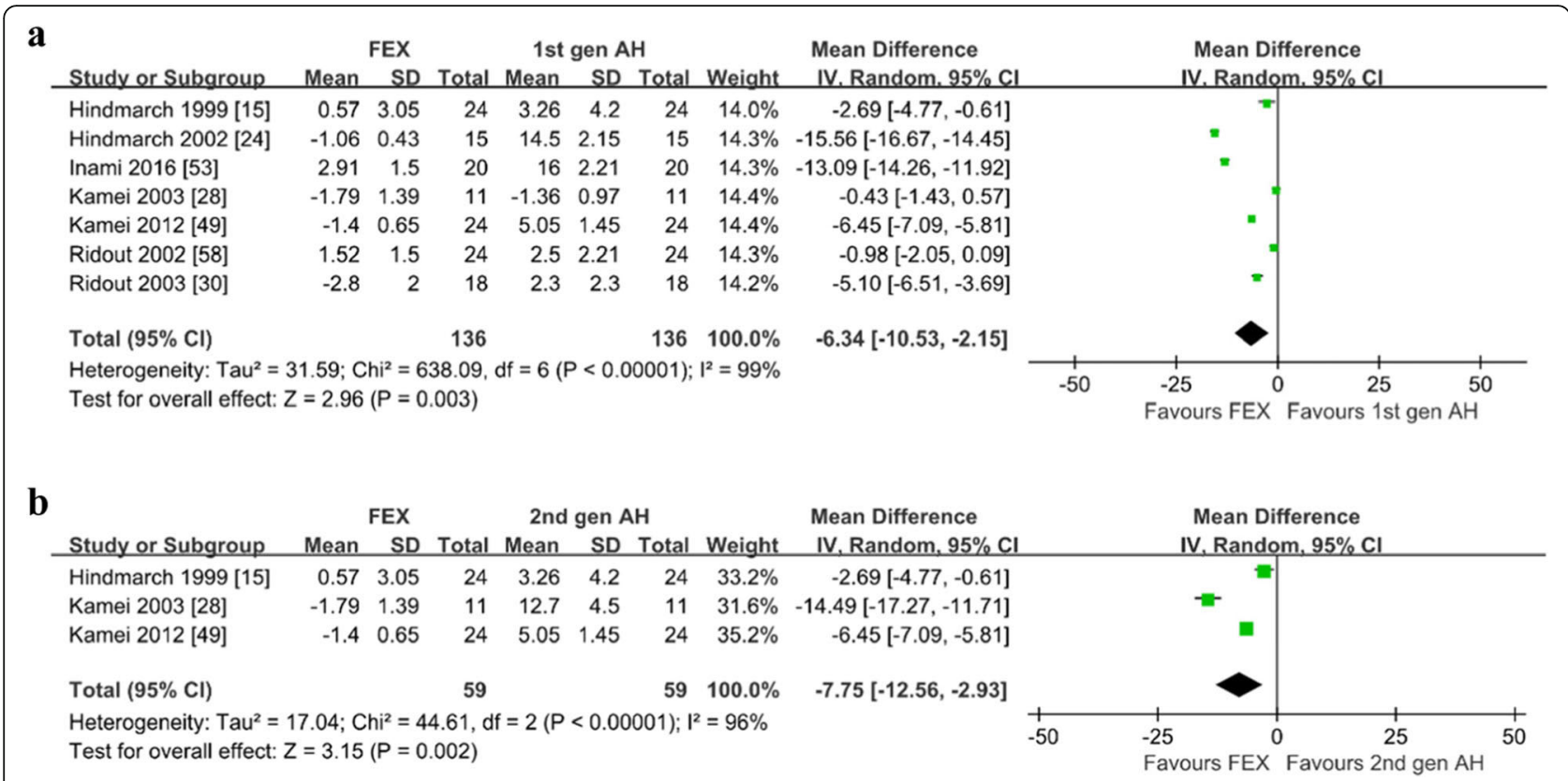

Fig. 9 Line analogue rating scales for sedation: a fexofenadine vs. the first-generation antihistamines; $\mathbf{b}$ fexofenadine vs. the second-generation antihistamines. 1st gen AH the first-generation antihistamines, 2nd gen AH second generation antihistamines

to $-1.35, P<0.0001)$. The heterogeneity may be generated from the inconsistent doses of fexofenadine and different antihistamines in each comparator. Sensitivity analysis showed the meta-analysis results were similar.

VAS of drowsiness. Three studies reported the VAS of drowsiness of subjects after taking fexofenadine $[19,56$, 59]. Of the 3 studies on healthy subjects, 2 compared with the first-generation antihistamines [19, 59], 2 compared with the second-generation antihistamines [56, 59], all compared with placebo $[19,56,59]$. The comparison between fexofenadine and the second-generation antihistamines was not pooled for meta-analysis because there was only 2 study included with a high heterogeneity. When compared with the first-generation antihistamines, as shown in Fig. 10, fexofenadine produced significantly less change of VAS of drowsiness (WMD = - 15.72; 95\% CI: -17.33 to $-14.11, P<0.00001)$. When compared with placebo, as shown in Additional file 10: Figure S10, the change of VAS of drowsiness were not different $(\mathrm{WMD}=7.18$; $95 \% \mathrm{CI}$ : -0.64 to $14.99, P=$
0.07). The heterogeneity may be generated from the inconsistent doses of fexofenadine. Sensitivity analysis showed the meta-analysis results were similar. Results summary is shown in Table 2 .

\section{Risk of Bias}

As shown in Additional file 1: Figure S1, most (82\%) of included studies had low risk of bias in random sequence generation. Twenty-two percent had low risk of bias in allocation concealment. Twenty-seven percent had low risk of bias in blinding of outcome assessment. All studies had low risk of bias in incomplete outcome data and selective reporting.

\section{Publication Bias}

Symmetry was shown in funnel plots when the safety of fexofenadine was compared to other antihistamines and placebo (Additional files 11: Figures S11, Additional file 12: Figure S12, Additional file 13: Figure S13, Additional file 14: Figure S14, Additional file 15: Figure S15,

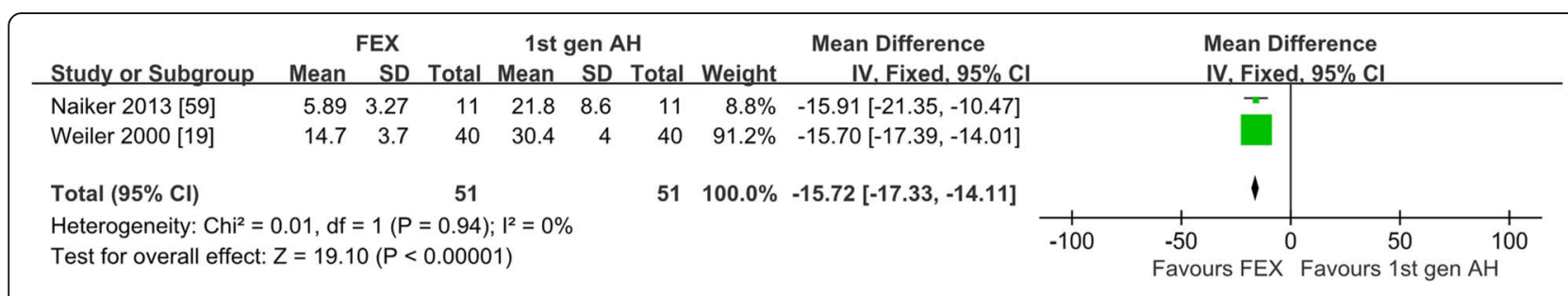

Fig. 10 Visual analogue drowsiness scores: fexofenadine vs. the first-generation antihistamines. 1st gen AH the first-generation antihistamines 


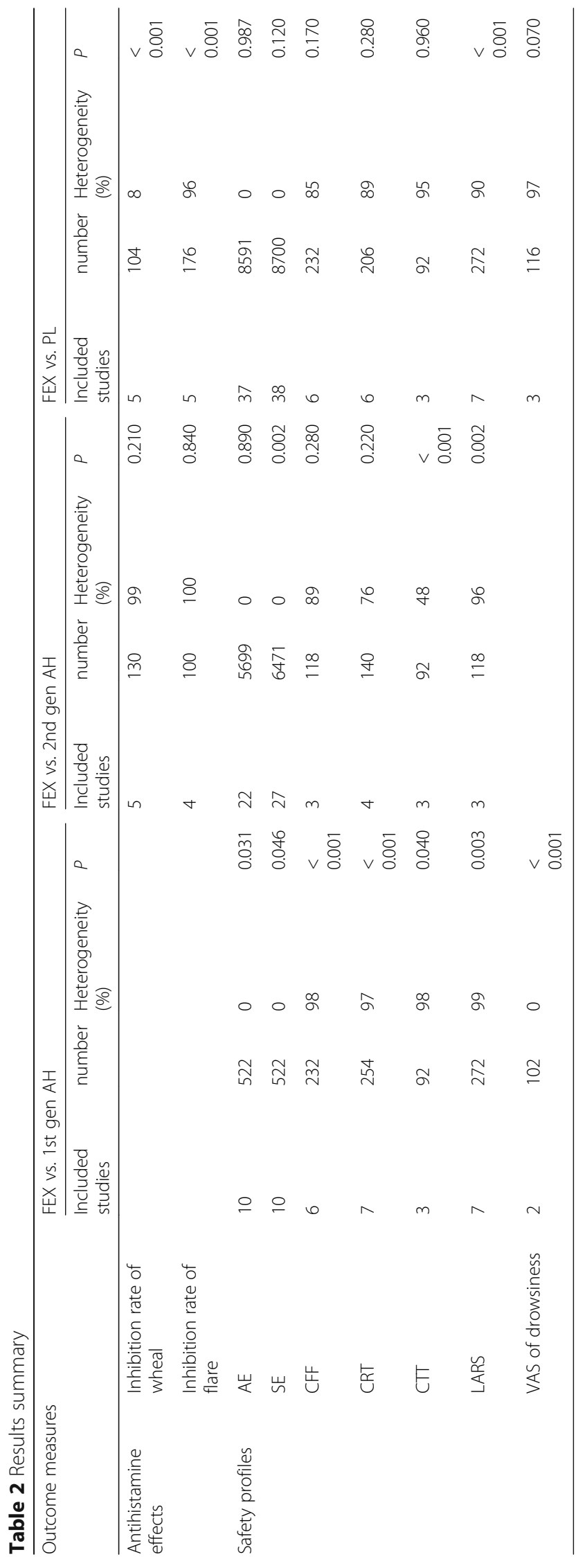


Additional file 16: Figure S16, Additional file 17: Figure S17, Additional file 18: Figure S18, Additional file 19: Figure S19), which means no publication bias in these analyses. A modest asymmetric funnel was shown when the antihistamine effect of fexofenadine was compared to other antihistamines and placebo (Additional files 11: Figure S11, Additional file 12: Figure S12, Additional file 13: Figure S13, Additional file 14: Figure S14, Additional file 15: Figure S15, Additional file 16: Figure S16, Additional file 17: Figure S17, Additional file 18: Figure S18, Additional file 19: Figure S19), which means no significant publication bias in these analyses.

\section{Discussion}

Based on our review of the literature, this is the first meta-analysis to assess the antihistamine effects and sedative effects of fexofenadine. Our meta-analysis indicates that fexofenadine has better safety profiles compared to the second-generation antihistamines. The antihistamine effects (the inhibition rate of histamineinduced wheal and flare) of fexofenadine were significantly higher than that of placebo and were not significantly different compared with the second-generation antihistamines.

Antihistamine effects that assessed by the inhibition rate of histamine-induced wheal and flare are important measurements to evaluate the efficacy of antihistamines in the treatment of allergic diseases [60]. Based on the pooled analysis, we find that the antihistamine effects and duration of fexofenadine are probably no worse than that of the second-generation antihistamines and more positive than that of placebo. Similarly to our study, a recent systematic review and meta-analysis showed that fexofenadine was effective on the treatment of nasal symptoms in patients with seasonal allergic rhinitis (SAR) [61]. On the contrast to that systematic review, our study includes all RCTs involving fexofenadine treatment that compared with other antihistamines or placebo by evaluating the inhibition rate of histamineinduced wheal and flare, not only just SAR RCTs. Therefore, fexofenadine that has positive antihistamine effects is suitable for most of the patients with indications requiring antihistamines. Of note, a study suggested that the inhibition rate of fexofenadine on histamine-induced wheal was lower than that of loratadine [36], while another study showed that fexofenadine had a significantly higher inhibition rate on histamine-induced wheal compared with loratadine [55]. The reason for this may be the different doses of fexofenadine $(60 \mathrm{mg} / \mathrm{d}$ and 180 $\mathrm{mg} / \mathrm{d})$ and the same dose of loratadine $(10 \mathrm{mg} / \mathrm{d})$ in the two study. Therefore, further studies are required to explore a more secure and effective dose of fexofenadine that compared with loratadine.
$\mathrm{AE}$ is closely related to drug damage to the body [62]. $\mathrm{AE}$ in included studies are as follows: headache, drowsiness, fatigue, upper respiratory infection, asthma, pharyngitis, dry mouth, cough, nausea, gastrointestinal pain, diarrhea, rash, epistaxis, sinusitis, back pain, leukopenia, etc. In the above $\mathrm{AE}$, headache is the most common $\mathrm{AE}$ in subjects who treated with fexofenadine. Overall, fexofenadine is well-tolerated and discontinuation owing to side effects generally occurs in $<5 \%$ of patients [63]. All the first-generation antihistamines and most of the second-generation antihistamines cause cardiotoxicity by inhibiting muscarinic cholinoreceptor (M-ChR), which can regulate heart rate, heart rhythm and cardiac muscle [64]. A previous study indicated that fexofenadine did not prolong QT interval and cause arrhythmia when it was used alone or combined with other drugs such as ketoconazole and erythromycin [65]. A dog model showed that fexofenadine was 600 times more affinity for $\mathrm{H} 1$ receptor than $\mathrm{M}-\mathrm{ChR}$, while desloratadine was only 5 times than M-ChR although it was also a new generation antihistamine, indicating that fexofenadine may have no cardiotoxicity [66]. This study find that there is no hepatotoxicity or cardiotoxicity related $\mathrm{AE}$ in subjects treated with fexofenadine, further supporting that fexofenadine may have no hepatotoxicity and cardiotoxicity. A systematic review and meta-analysis showed that there was no significant difference of $\mathrm{AE}$ frequency between fexofenadine and placebo in patients with SAR [61]. Consistently in the respect of $\mathrm{AE}$, our study indicates that the safety profile of fexofenadine is more positive than that of the first-generation antihistamines and similar to the second-generation antihistamines and placebo.

Sedative effect is one of the most concerned issue of $\mathrm{AE}$ [67]. The current study indicates that the risk of fexofenadine on sedative effects is lower than that of the firstgeneration antihistamines and the second-generation antihistamines, and similar to placebo. A previous study showed that fexofenadine may have no sedative effect or only have mild sedative effects since fexofenadine could not pass the blood-brain barrier [68]. Our study demonstrates that the risk of fexofenadine on sedative effects was lower than that of the first-generation antihistamines and the second-generation antihistamines and even has been as low as placebo. A recent meta-analysis showed that levocetirizine had a mild sedative effects although it was a new generation antihistamine [69]. According to our study, fexofenadine with no sedative effect is more worthy of recommendation among the new generation antihistamines. Based on the fact that fexofenadine may have no sedative effect, an expert consensus in the United States recommended National Aeronautics and Space Administration (NASA) to authorize pilots to use fexofenadine if necessary [70]. 
The cognitive/psychomotor function is another important relevant issue of $\mathrm{AE}$ [71]. This study suggests that fexofenadine has less cognitive/psychomotor impairment compared with both of the first-generation antihistamines and the second-generation antihistamines. In addition, the cognitive/psychomotor impairment of fexofenadine is similar to placebo. A recent systematic review suggested that fexofenadine was ranked as the least psychomotor impairment antihistamines compared with all other antihistamines in Japanese market [72]. In contrast to that systematic review, our study included antihistamines used worldwide. Furthermore, we added a comparison between fexofenadine and placebo. The results further identify the fact that fexofenadine may have no cognitive/psychomotor impairment. As for CFF, we find that fexofenadine has a positive information processing capability compared with promethazine. Similarly, a study showed that the information processing capability after treating with fexofenadine $120 \mathrm{mg} / \mathrm{d}$ or $60 \mathrm{mg} / \mathrm{d}$ was better than olopatadine $10 \mathrm{mg} / \mathrm{d}$ or $5 \mathrm{mg} / \mathrm{d}$ $[28,49]$. But another study demonstrated that the information processing capability after treating with fexofenadine $120 \mathrm{mg} / \mathrm{d}$ was worse than that of loratadine $10 \mathrm{mg} /$ $\mathrm{d}$ [15]. The above discordance may be attributed to the differences of washout period. Only 4 days applied in study of Hindmarch may result in insufficient drug clearance, which affected the reliability of its result. Therefore, although fexofenadine has marginal cognitive/psychomotor impairment compared with the second-generation antihistamines, the comparison on cognitive/psychomotor function between fexofenadine and individual second-generation antihistamine remains to be further explored.

There are several potential limitations in this study. The setting of subgroups regarding dose/duration/type of antihistamines was unavailable because of limited studies. The comparison of antihistamine effects and cognitive/psychomotor function were lack of large sample RCTs, which may increase the risk of bias. The way to obtain outcome measures such as the frequency of $\mathrm{AE}$ and sedative effects was different.

\section{Conclusions}

Fexofenadine has a positive antihistamine effect, which is probably no worse than the second-generation antihistamines. Fexofenadine probably has a favorable safety profile, which is more likely better than that of the firstgeneration antihistamines. There is lack of data to support that fexofenadine has a better overall safety profile compared to the second-generation antihistamines, however, some presently available evidence on sedative effects and certain aspects of cognitive/psychomotor function favors fexofenadine. Therefore, fexofenadine may be worthy of recommendation for safety related workers. However, more multicenter, large sample, longterm follow-up and well-designed head-to-head trials are required to the further understanding of the efficacy and safety of fexofenadine.

\section{Supplementary information}

Supplementary information accompanies this paper at https://doi.org/10. 1186/s40360-019-0363-1.

Additional file 1: Figure S1. Risk of bias: a risk of bias summary; b risk of bias graph.

Additional file 2. Figure S2. Forest plot of wheal for FEX vs. PL. Additional file 3. Figure S3. Forest plot of flare for FEX vs. PL. Additional file 4. Figure S4. Forest plot of AE for FEX vs. PL. Additional file 5. Figure S5. Forest plot of SE for FEX vs. PL. Additional file 6. Figure S6. Forest plot of CFF for FEX vs. PL. Additional file 7. Figure S7. Forest plot of CRT for FEX vs. PL. Additional file 8. Figure S8. Forest plot of CTT for FEX vs. PL. Additional file 9. Figure S9. Forest plot of LARS for FEX Vs. PL. Additional file 10. Figure S10. Forest plot of VAS of drowsiness for FEX vs. PL.

Additional file 11: Figure S11. Funnel plot of wheal: a fexofenadine vs. the second-generation antihistamines; b fexofenadine vs. placebo.

Additional file 12: Figure S12. Funnel plot of flare: a fexofenadine vs. the second-generation antihistamines; $b$ fexofenadine vs. placebo.

Additional file 13: Figure S13. Funnel plot of adverse events: a fexofenadine vs. the first-generation antihistamines; $b$ fexofenadine vs. the second-generation antihistamines; c fexofenadine vs. placebo.

Additional file 14: Figure S14. Funnel plot of sedative effects: a fexofenadine vs. the first-generation antihistamines; $b$ fexofenadine vs. the second-generation antihistamines; c fexofenadine vs. placebo.

Additional file 15: Figure S15. Funnel plot of CFF: a fexofenadine vs. the first-generation antihistamines; $b$ fexofenadine vs. the secondgeneration antihistamines; c fexofenadine vs. placebo.

Additional file 16: Figure S16. Funnel plot of CRT: a fexofenadine vs. the first-generation antihistamines; $b$ fexofenadine vs. the secondgeneration antihistamines; c fexofenadine vs. placebo.

Additional file 17: Figure S17. Funnel plot of CTT: a fexofenadine vs. the first-generation antihistamines; $b$ fexofenadine vs. the secondgeneration antihistamines; c fexofenadine vs. placebo.

Additional file 18: Figure S18. Funnel plot of LARS: a fexofenadine vs. the first-generation antihistamines; $b$ fexofenadine vs. the secondgeneration antihistamines; c fexofenadine vs. placebo.

Additional file 19: Figure S19. Funnel plot of VAS of drowsiness: a fexofenadine vs. the first-generation antihistamines; $b$ fexofenadine vs. placebo.

\section{Abbreviations}

FEX: Fexofenadine; EBA: Ebastine, LOR: Loratadine; CHL: Chlorpheniramine. CET: Cetirizine, BEP: Bepotastine; EPI: Epinastine,; TER: Terfenadine, MI: Mizolastine, BIL bilastine, ${ }_{\text {, PR: Promethazine, }}$ DI: Diphenhydramine,

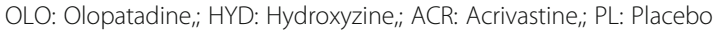

\section{Acknowledgments}

We are grateful to the authors of the primary studies included in this metaanalysis.

\section{Authors' contributions}

$\mathrm{CH}$ and $\mathrm{ZJ}$ performed the literature searches, selected the studies, analyzed the data, and wrote the manuscript draft. JW and $Y L$ aided in the data analysis. HP designed the study and revised manuscript. All authors have read and approved the manuscript, and ensure that this is the case. 


\section{Funding}

This work was supported by National Natural Science Foundation of China (No. 81300817), who participated in the design of the study. This work was supported by Natural Science Foundation of Guangdong Province (No. 2018A0303130338), who participated in obtain of full text and the collection, analysis, and interpretation of data. This work was also supported by Science and Technology Project Foundation of Guangzhou (No. 201604020106), who participated in obtain of software for Meta analysis and writing the manuscript.

\section{Availability of data and materials}

All data generated or analyzed during this study are included in this published article. Additional information may be requested directly from the study authors.

\section{Ethics approval and consent to participate}

This article does not contain any studies with human participants or animals performed by any of the authors.

\section{Consent for publication}

Not applicable.

\section{Competing interests}

The authors declare that they have no competing interests.

\section{Author details}

'Department of Otolaryngology Head and Neck Surgery, General Hospital of Southern Theatre Command of PLA, Guangzhou 510010, China. ${ }^{2}$ Department of Otolaryngology Head and Neck Surgery, Shenzhen University General Hospital, Shenzhen 518055, China. ${ }^{3}$ Department of Pharmacy, General Hospital of Southern Theatre Command of PLA, Guangzhou 510010, China. ${ }^{4}$ Integrated Hospital of Traditional Chinese Medicine, Southern Medical University, Guangzhou 510315, China. ${ }^{5}$ Cancer Center, Southern Medical University, Guangzhou 510315, China.

Received: 7 December 2018 Accepted: 2 October 2019

Published online: 29 November 2019

\section{References}

1. Brozek G, Lawson J, Szumilas D, Zejda J. Increasing prevalence of asthma, respiratory symptoms, and allergic diseases: four repeated surveys from 1993-2014. Respir Med. 2015;109(8):982-90.

2. Baiardini I, Braido F, Brandi S, Canonica GW. Allergic diseases and their impact on quality of life. Ann Allergy Asthma Immunol. 2006;97(4):419-28.

3. Zuberbier T, Lotvall J, Simoens S, Subramanian SV, Church MK. Economic burden of inadequate management of allergic diseases in the European Union: a GA (2) LEN review. Allergy. 2014:69(10):1275-9.

4. Horak F, Stubner UP. Comparative tolerability of second generation antihistamines. Drug Saf. 1999;20(5):385-401.

5. Sorkin EM, Heel RC. Terfenadine. A review of its pharmacodynamic properties and therapeutic efficacy. Drugs. 1985;29(1):34-56.

6. Chen C. Some pharmacokinetic aspects of the lipophilic terfenadine and zwitterionic fexofenadine in humans. Drugs. 2007;8(5):301-14.

7. Yanai K, Rogala B, Chugh K, Paraskakis E, Pampura AN, Boev R. Safety considerations in the management of allergic diseases: focus on antihistamines. Curr Med Res Opin. 2012;28(4):623-42.

8. Higgins J, Green S. Cochrane handbook for systematic reviews of interventions version 5.1.0 [Updated March 2011]. The Cochrane Collaboration. 2011.

9. Simons FE, Simons KJ. Peripheral H1-blockade effect of fexofenadine. Ann Allergy Asthma Immunol. 1997;79(6):530-2.

10. Bronsky EA, Falliers CJ, Kaiser HB, Ahlbrandt R, Mason JM. Effectiveness and safety of fexofenadine, a new nonsedating $\mathrm{H1}$-receptor antagonist, in the treatment of fall allergies. Allergy Asthma Proc. 1998;19(3):135-41.

11. Albert F, Finn J, Kaplan AP, Fretwell R, Qu R, Long J, et al. A double-blind, placebo-controlled trial of fexofenadine $\mathrm{HCl}$ in the treatment of chronic idiopathic urticaria. J Allergy Clin Immunol. 1999:103:1071-8.

12. Ballmer-Weber BK, Gex-Collet C, Wuthrich B. Inhibition of histamine or allergen-induced wheals by a single dose of acrivastine, fexofenadine or cetirizine. J Investig Allergol Clin Immunol. 1999;9(6):351-5.
13. Casale TB, Andrade C, Qu R. Safety and efficacy of once-daily fexofenadine $\mathrm{HCl}$ in the treatment of autumn seasonal allergic rhinitis. Allergy Asthma Proc. 1999;20(3):193-8.

14. Grant JA, Danielson L, Rihoux J-P, Delvos C. A double-blind, singledose, crossover comparison of cetirizine, ebastine, epinastine, fexofenadine, terfenadine, and loratadine versus placebo suppression of histamine-induced wheal and flare response for $24 \mathrm{~h}$ in. Allergy. 1999;54:700-7.

15. Hindmarch I, Shamsi Z, Stanley N, Fairweather DB. A double-blind, placebocontrolled investigation of the effects of fexofenadine, loratadine and promethazine on cognitive and psychomotor function. Blackwell Science Ltd Br J Clin Pharmacol. 1999;48:200-6.

16. Howarth PH, Stern MA, Roi L, Reynolds R, Bousquet J. Double-blind, placebo-controlled study comparing the efficacy and safety of fexofenadine hydrochloride (120 and $180 \mathrm{mg}$ once daily) and cetirizine in seasonal allergic rhinitis. J Allergy Clin Immunol. 1999;104(5):927-33.

17. CAUWENBERGE PV, JUNIPER EF, GROUP TSSI. Comparison of the efficacy, safety and quality of life provided by fexofenadine hydrochloride $120 \mathrm{mg}$, loratadine $10 \mathrm{mg}$ and placebo administered once daily for the treatment of seasonal allergic rhinitis. Clin Exp Allergy. 2000;30:891-9.

18. Prenner BM, Capano D, Harris AG. Efficacy and tolerability of loratadine versus fexofenadine in the treatment of seasonal allergic rhinitis: a doubleblind comparison with crossover treatment of nonresponders. Clin Ther. 2000;22(6):760-9.

19. Weiler JM, Bloomfield JR, Woodworth GG, Grant AR, Layton TA, Brown TL, et al. Effects of fexofenadine, diphenhydramine, and alcohol on driving performance. A randomized, placebo-controlled trial in the lowa driving simulator. Ann Intern Med. 2000;132(5):354-63.

20. Horak F, Stübner P, Zieglmayer R, Kavina A, Vos CD, Burtinb B, et al. Controlled comparison of the efficacy and safety of cetirizine $10 \mathrm{mg}$ o.d. and fexofenadine $120 \mathrm{mg}$ o.d. in reducing symptoms of seasonal allergic rhinitis. Int Arch Allergy Immunol. 2001;125:73-9.

21. Kaiser HB, Rooklin A, Spangler D, Capano D. Efficacy of Loratadine compared with fexofenadine or placebo for the treatment of seasonal allergic rhinitis. Clin Drug Invest. 2001;21(8):571-8.

22. Purohit A, Duvernelle C, Melac M, Pauli G, Frossard N. Twenty-four hours of activity of cetirizine and fexofenadine in the skin. Ann Allergy Asthma Immunol. 2001;86(4):387-92

23. Grant JA, Riethuisen J-M, Ba M, DeVos C. A double-blind, randomized, single-dose, crossover comparison of levocetirizine with ebastine, fexofenadine, loratadine, mizolastine, and placebo: suppression of histamine-induced wheal-and-flare response during 24 hours in healthy male subjects. Ann Allergy Asthma Immunol. 2002;88:190-7.

24. Hindmarch I, Shamsi Z, Kimber S. An evaluation of the effects of high-dose fexofenadine on the central nervous system: a double-blind, placebocontrolled study in healthy volunteers. Clin Exp Allergy. 2002;32(1):133-9.

25. Barbanoj MJ, Antonijoan RM, Garcia-Gea C, Morte A, Gich I, Gispert J, et al. A study comparing the inhibitory effects of single and repeated oral doses of ebastine and fexofenadine against histamine-induced skin reactivity. Int Arch Allergy Immunol. 2003;132(3):263-7.

26. Hampel F, Ratner P, Mansfield L, Meeves S, Liao Y, Georges G. Fexofenadine hydrochloride, $180 \mathrm{mg}$, exhibits equivalent efficacy to cetirizine, $10 \mathrm{mg}$, with less drowsiness in patients with moderate-to-severe seasonal allergic rhinitis. Ann Allergy Asthma Immunol. 2003;91:354-61.

27. Handa S, Dogra S, Kumar B. Comparative efficacy of cetirizine and fexofenadine in the treatment of chronic idiopathic urticaria. J Dermato Treat. 2003;15(1):55-7.

28. Kamei H, Noda Y, Ishikawa K, Senzaki K, Muraoka I, Hasegawa Y, et al. Comparative study of acute effects of single doses of fexofenadine, olopatadine, $\mathrm{d}$-chlorpheniramine and placebo on psychomotor function in healthy volunteers. Human Psychopharmacol. 2003;18(8):611-8.

29. Mansfield L, Mendoza C, Flores J, Meeves SG. Effects of fexofenadine, diphenhydramine, and placebo on performance of the test of variables of attention (TOVA). Ann Allergy Asthma Immunol. 2003; 90(5):554-9.

30. Ridout F, Shamsi Z, Meadows R, Johnson S, Hindmarch I. A single-center, randomized, double-blind, placebo-controlled, crossover investigation of the effects of fexofenadine hydrochloride $180 \mathrm{mg}$ alone and with alcohol, with hydroxyzine hydrochloride $50 \mathrm{mg}$ as a positive internal control, on aspects of cognitive and psychomotor function related to driving a car. Clin Ther. 2003;25(5):1518-38 
31. Simons F, Semus M, Goritz S, Simons K. H1-antihistaminic activity of cetirizine and fexofenadine in allergic children. Pediatr Allergy Immunol. 2003;14(3):207-11.

32. Wahn U, Meltzer EO, Finn AF, Kowalski ML, Decosta P, Hedlin G, et al. Fexofenadine is efficacious and safe in children (aged 6-11 years) with seasonal allergic rhinitis. J Allergy Clin Immunol. 2003;111(4):763-9.

33. Day JH, Briscoe MP, Rafeiro E, Hewlett D Jr, Chapman D, Kramer B. Randomized double-blind comparison of cetirizine and fexofenadine after pollen challenge in the environmental exposure unit: duration of effect in subjects with seasonal allergic rhinitis. Allergy Asthma Proc. 2004;25(1):59-68.

34. Horak F, Zieglmayer PU, Zieglmayer R, Kavina A, Lemell P. Levocetirizine has a longer duration of action on improving total nasal symptoms score than fexofenadine after single administration. Br J Clin Pharmacol. 2004;60(1):24-31.

35. Purohit A, N'Gom AS, Deslandes B, Pauli G, Frossard N. Similar rapid onset of action and magnitude of effect of fexofenadine and cetirizine as assessed by inhibition of histamine-induced wheal-and-flare reaction. Ann Allergy Asthma Immunol. 2004:93(6):562-7.

36. Boyle J, Ridout F, Meadows R, Johnsen S, Hindmarch I. Suppression of the histamine-induced wheal and flare response by fexofenadine $\mathrm{HCl} 60 \mathrm{mg}$ twice daily, loratadine $10 \mathrm{mg}$ once daily and placebo in healthy Japanese volunteers. Curr Med Res Opin. 2005:21(9):1495-503.

37. Day JH, Briscoe MP, Rafeiro E, Ratz JD, Ellis AK, Frankish CW, et al. Comparative efficacy of cetirizine and fexofenadine for seasonal allergic rhinitis, 5-12 hours postdose, in the environmental exposure unit. Allergy Asthma Proc. 2005;26(4):275-82.

38. Hyo S, Fujieda S, Kawada R, Kitazawa S, Takenaka H. The efficacy of shortterm administration of 3 antihistamines vs placebo under natural exposure to Japanese cedar pollen. Ann Allergy Asthma Immunol. 2005;94(4):457-64

39. Schapowal A, Study G. Treating intermittent allergic rhinitis: a prospective, randomized, placebo and antihistamine-controlled study of butterbur extract Ze 339. Phytother Res. 2005;19(6):530-7.

40. Tsuda H, Takiwaki H. Comparison of on-site and photographic evaluations of the suppressive effects of cetirizine, loratadine, and fexofenadine on skin response to histamine lontophoresis: a double-blind, crossover study in healthy volunteers. Curr Ther Res, Clin Exp. 2005;66(4):307-22.

41. Berger WE, Lumry WR, Meltzer EO, Pearlman DS. Efficacy of desloratadine, 5 $\mathrm{mg}$, compared with fexofenadine, $180 \mathrm{mg}$, in patients with symptomatic seasonal allergic rhinitis. Allergy Asthma Proc. 2006;27(3):214-23.

42. Berkowitz RB, Braker S, Lutz C, Jones P, Meeves S, Qiu C, et al. Efficacy of fexofenadine in the prophylactic control of cat allergen-induced allergic rhinitis. Ann Allergy Asthma Immunol. 2006;96(2):327-33.

43. Boyle J, Eriksson M, Stanley N, Fujita T, Kumagi Y. Allergy medication in Japanese volunteers: treatment effect of single doses on nocturnal sleep architecture and next day residual effects. Curr Med Res Opin. 2006;22(7): 1343-51.

44. Okubo K, Gotoh M. Inhibition of the antigen provoked nasal reaction by second-generation antihistamines in patients with Japanese cedar pollinosis. Allergol Int. 2006;55(3):261-9.

45. Hampel FC, Kittner B, van Bavel JH. Safety and tolerability of fexofenadine hydrochloride, 15 and $30 \mathrm{mg}$, twice daily in children aged 6 months to 2 years with allergic rhinitis. Ann Allergy Asthma Immunol. 2007;99(6):549-54.

46. Milgrom H, Kittner B, Lanier R, Hampel FC. Safety and tolerability of fexofenadine for the treatment of allergic rhinitis in children 2 to 5 years old. Ann Allergy Asthma Immunol. 2007;99(4):358-63.

47. Kaiser HB, Gopalan G, Chung W. Loratadine provides early symptom control in seasonal allergic rhinitis. AllergyAsthma Proc. 2008;29(6):654-8.

48. Horak F, Zieglmayer P, Zieglmayer R, Lemell P. The effects of bilastine compared with cetirizine, fexofenadine, and placebo on allergen-induced nasal and ocular symptoms in patients exposed to aeroallergen in the Vienna challenge chamber. Inflamm Res. 2010;59(5):391-8.

49. Kamei H, Isaji A, Noda Y, Ishikawa K, Senzaki K, Yamada K, et al. Effects of single therapeutic doses of promethazine, fexofenadine and olopatadine on psychomotor function and histamine-induced wheal- and flare-responses: a randomized double-blind, placebo-controlled study in healthy volunteers. Arch Dermatol Res. 2012;304(4):263-72.

50. Ramesh LA. A comparative study of fexofenadine with CHLORPHENIRAMINE maleate and betamethasone in allergic rhinitis. Int J Pharm Bio Sci. 2013; 4(1):128-34.

51. Schoepke N, Church MK, Maurer M. The inhibition by levocetirizine and fexofenadine of the histamine-induced wheal and flare response in healthy Caucasian and Japanese volunteers. Acta Derm Venereol. 2013;93(3):286-93.
52. Hashiguchi K, Wakabayashi Kl, Togawa M, Saito A, Okubo K. Therapeutic effect of bilastine in Japanese cedar pollinosis using an artificial exposure chamber (OHIO chamber). Allergol Int. 2016;66(1):123-31.

53. Inami $A$, Matsuda $R$, Grobosch $T$, Komamura $H$, Takeda $K$, Yamada $Y$, et al. A simulated car-driving study on the effects of acute administration of levocetirizine, fexofenadine, and diphenhydramine in healthy Japanese volunteers. Human Psychopharmacol. 2016;31(3):167-77.

54. Okubo K, Gotoh M, Asako M, Nomura Y, Togawa M, Saito A, et al. Efficacy and safety of bilastine in Japanese patients with perennial allergic rhinitis: a multicenter, randomized, double-blind, placebo-controlled, parallel-group phase III study. Allergol Int. 2016;66(1):97-105.

55. Simons S, Gu S. Clinical pharmacology of $\mathrm{H1}$-antihistamines in the skin. J Allergy Clin Immunol. 2002;110(5):777-83.

56. Takahashi H, Ishida-Yamamoto A, lizuka H. Effects of bepotastine, cetirizine, fexofenadine, and olopatadine on histamine-induced wheal-and flareresponse, sedation, and psychomotor performance. Clin Exp Dermatol. 2004;29(5):526-32.

57. Larbig M, Burtin B, Martin L, Stamm H, Luettig B, Hohlfeld JM, et al. Facial thermography is a sensitive tool to determine antihistaminic activity: comparison of levocetirizine and fexofenadine. Br J Clin Pharmacol. 2006; 62(2):158-64.

58. Ridout F, Hindmarch I. The effects of acute doses of fexofenadine, promethazine, and placebo on cognitive and psychomotor function in healthy Japanese volunteers. Ann Allergy Asthma Immunol. 2002:90(4):404-10.

59. Naicker P, Anoopkumar-Dukie S, Grant GD, Kavanagh JJ. The effects of antihistamines with varying anticholinergic properties on voluntary and involuntary movement. Clin Neurophysiol. 2013;124(9):1840-5.

60. Monroe EW, Daly AF, Shalhoub RF. Appraisal of the validity of histamineinduced wheal and flare to predict the clinical efficacy of antihistamines. $J$ Allergy Clin Immunol. 1997;99(2):S798-806.

61. Compalati E, Baena-Cagnani R, Penagos M, Badellino H, Braido F, Gomez RM, et al. Systematic review on the efficacy of fexofenadine in seasonal allergic rhinitis: a meta-analysis of randomized, double-blind, placebocontrolled clinical trials. Int Arch Allergy Immunol. 2011;156(1):1-15.

62. Nicholls J, MacKenzie C, Braund R. Preventing drug-related adverse events following hospital discharge: the role of the pharmacist. Integr Pharm Res Pract. 2017:6:61-9.

63. Smith SM, Gums JG. Fexofenadine: biochemical, pharmacokinetic and pharmacodynamic properties and its unique role in allergic disorders. Expert Opin Drug Metab Toxicol. 2009;5(7):813-22.

64. DuBuske LM. Second-generation antihistamines: the risk of ventricular arrhythmias. Clin Ther. 1999:21(2):281-95.

65. Mazier C, Jaouen M, Sari MA, Buisson D. Microbial oxidation of terfenadine and ebastine into fexofenadine and carebastine. Bioorg Med Chem Lett. 2004;14(21):5423-6. https://doi.org/10.1016/j.bmcl.2004.07.076

66. Simons FE, Silver NA, Gu X, Simons KJ. Skin concentrations of H1-receptor antagonists. J Allergy Clin Immunol. 2001;107(3):526-30.

67. Saunders R, Davis JA, Kranke P, Weissbrod R, Whitaker DK, Lightdale JR. Clinical and economic burden of procedural sedation-related adverse events and their outcomes: analysis from five countries. Ther Clin Risk Manag. 2018;14:393-401

68. Hiraoka K, Tashiro M, Grobosch T, Maurer M, Oda K, Toyohara J, et al. Brain histamine $\mathrm{H} 1$ receptor occupancy measured by PET after oral administration of levocetirizine, a non-sedating antihistamine. Expert Opin Drug Saf. 2015; 14(2):199-206.

69. Snidvongs K, Seresirikachorn K, Khattiyawittayakun L, Chitsuthipakorn W. Sedative effects of Levocetirizine: a systematic review and Meta-analysis of randomized controlled studies. Drugs. 2017;77(2):175-86.

70. DuBuske L. Pharmacokinetics/pharmacodynamics and psychomotor performance aspects of antihistamine therapies. Clin Appl Immunol Rev. 2001:1:277-89.

71. Riedel WJ, Mehta MA, Unema PJ. Human cognition assessment in drug research. Curr Pharm Des. 2006;12(20):2525-39.

72. Isomura T, Kono T, Hindmarch I, Kikuchi N, Murakami A, Inuzuka K, et al. Central nervous system effects of the second-generation antihistamines marketed in Japan--review of inter-drug differences using the proportional impairment ratio (PIR). PLoS One. 2014;9(12):e114336.

\section{Publisher's Note}

Springer Nature remains neutral with regard to jurisdictional claims in published maps and institutional affiliations. 